Alma Mater Studiorum - Università di Bologna

Scuola di Scienze

Dipartimento di Fisica e Astronomia

Corso di Laurea in Fisica

\title{
Linear Irreversible Thermodynamics
}

Relatore:

Prof. Loris Ferrari

Presentata da:

Brenno J. S. P. De Lucca

Anno Accademico 2019/2020 
Turning and turning in a widening gyre

The falcon cannot hear the falconer;

Things fall apart; the centre cannot hold;

Mere anarchy is loosed upon the world 


\section{Abstract}

In questa tesi tratteremo il problema di costruire una teoria termodinamica per trasformazioni su un sistema passante per stati di non-equilibrio. Cercando di generalizzare a sistemi che non sono all'equilibrio, rilasseremo la richiesta che siano in equilibrio globalmente. Lo stato termodinamico sarà univocamente determinato da un insieme di parametri termodinamici definiti localmente, della stessa natura e significato fisico dei parametri usati nella termodinamica classica. Le molteplici assunzioni necessarie al fine di avere una teoria mesoscopica comunque predittiva verranno giustificate a posteriori, quando possibile, in base alle predizioni che da tale modello nasceranno. In particolare ci concentreremo sugli effetti termoelettrici di Thompson, Seebeck e Peltier, esempi storici di grande rilevanza nel campo della termodinamica del non-equilibrio. 


\section{Contents}

$\begin{array}{ll}\text { Introduction } & 4\end{array}$

1 The First Law of Thermodynamics $\quad 6$

1.1 Local Equilibrium . . . . . . . . . . . . . . . . . . . . . 6

1.2 Conservation of Mass . . . . . . . . . . . . . . . . . 7

1.3 Equations of Motion . . . . . . . . . . . . . . . . . . . . . . . . . . . . . . . . . 10

1.4 Conservation of Energy . . . . . . . . . . . . . . . . . . . . . 13

1.5 Local Expression of the First Law . . . . . . . . . . . . . . . . . 15

2 The Second Law of Thermodynamics 17

2.1 Local Expression of the Second Law . . . . . . . . . . . . . . . . . . . 17

2.2 Entropy Flux Density and Production Rate . . . . . . . . . . . . . 19

3 Linear Phenomenological Equations 23

3.1 Entropy Production and The Linear Laws . . . . . . . . . . . . . . . 23

3.2 The Curie Principle and Onsanger Reciprocal Relations . . . . . . . . . . 25

3.2.1 Magnetic Fields and Rotating Systems . . . . . . . . . . . . . . . 31

4 Application to Thermoelectric Phenomena 32

4.1 The Seebeck, Peltier and Thompson Effects . . . . . . . . . . . . . . . 32

4.2 Proof of Thompson's Relations . . . . . . . . . . . . . . . . 36

$\begin{array}{ll}\text { Appendix } & 46\end{array}$

$\begin{array}{ll}\text { Bibliography } & 49\end{array}$ 


\section{Introduction}

Although the general and consistent formulation of a thermodynamic theory for systems arbitrarily far from equilibrium is still a work in progress, the theory of linear irreversible thermodynamics is well established.

Classical statistical mechanics, first elaborated by L. Boltzmann and J. W. Gibbs managed to ground phenomenological quantities such as Temperature, Entropy and Heat, and the relations linking them, in the statistical dynamical microscopic description of a system.

Unfortunately, the predictive power of their theory cannot be turned to the study of systems out of equilibrium, that is, to the study of irreversible processes, yet this is the state in which a vast number of interesting systems do dwell, and in particular all living organisms.

The obstacles faced by theorists who have attempted to bridge the gap are significant. The foremost conceptual problem appears to be the actual definition of thermodynamic quantities, such as temperature, entropy and heat. The requirement that a system be in equilibrium for their definition to be coherent seems, a priori, to cripple any attempt to build a thermodynamic description of an irreversible process.

Having said that we shall see how, if done carefully, a construction of a theory of nonequilibrium thermodynamics along classical lines is possible. In particular we shall see how to skirt the definition problem of thermodynamic variables, provided that systems be sufficiently near to equilibrium that a local notion of equilibrium can be assigned to each point in space.

To this end, we shall work in an analogous fashion to the continuum model of fluid dynamics by defining local flows and fluxes of thermodynamic quantities and developing balance equations governing their evolution.

From the explicit expression for the rate of change of the entropy we shall observe that, inside an arbitrary volume element, the entropy of the volume can change for two reasons: entropy can flow into or out of the volume in the form of a flux, or it can be produced by a source term, due to irreversible phenomena happening within the volume.

A discussion will ensue on the consequences two important theorems in the theory of local equilibrium thermodynamics, namely due to Onsager and Curie, which make use of the system's spatial (discrete or continuous) symmetries and the invariance of the 
equations of motion under time reversals, to simplify considerably the bulk of differential equations describing the system's evolution.

In the final chapter we shall put everything together and see how the theory holds up when applied to the Peltier and Seebeck effects, two thermoelectric phenomena, which constituted considerable historic landmarks for the theory of linear irreversible thermodynamics, and especially, for the Onsanger relations. 


\section{Chapter 1}

\section{The First Law of Thermodynamics}

\subsection{Local Equilibrium}

To generalize the theory of equilibrium thermodynamics to treat irreversible phenomena we require that the system still retains a notion of equilibrium locally. To present a concrete example that immediately highlights the similarities with continuum fluid mechanics, let's consider a heterogeneous fluid situated in a region of space $\Omega$.

Although a constant value for all the state variables cannot be defined in the entire mixture, we suppose that it is composed of many regions, sufficiently small and each of volume $\mathrm{d} V$, such that a well-defined value of temperature $T$, pressure $P$, density $\rho$, velocity vector $\vec{v}$ and chemical potential $\mu^{1}$ can be ascribed to each at a certain time.

In formal terms, we ask that the system must be characterized by a length scale $L$, such that:

$$
\begin{gathered}
L \sim \frac{\gamma}{|\nabla \gamma(x, y, z)|} \\
\mathrm{d} V \sim L^{3}
\end{gathered}
$$

Where $\gamma$ is any one of the parameters of interest in the local-state description of the system. The volumes must therefore not exceed this characteristic volume, nor become too small, when the number of particles contained becomes statistically intractable.

This necessary working hypothesis is actually rather restrictive, as we shall see later in more detail. For now, it suffices to say that, analogously to the continuum model of fluid mechanics, the partition volumes dV must satisfy the condition that, for any state variable we wish to examine, the difference in value that a parameter takes between contiguous volumes must be negligibly small.

\footnotetext{
${ }^{1}$ Naturally in the case of a mixture the chemical potential, density and velocity will refer to the various $\mu_{k}, \rho_{k}$ and $\vec{v}_{k}$ of each chemical species $k$, and the total pressure will be, by Dalton's law, equal to the sum of the partial pressures.
} 
In addition, if we are to construct a thermodynamic theory locally, that is, in partial differential equation form, the state variables must be differentiable functions of the position $x \in \Omega$ and of time $t \in I$, and therefore at least class $C^{1}(\Omega \times I)$.

These are clearly stringent demands, for they imply that the system as a whole be sufficiently near equilibrium that there be almost no discontinuities ${ }^{2}$ in the composition of the system. Moreover the state functions, now defined as densities, will be assumed to have the same form as the canonical state functions in classical thermodynamics. This fact of not being arbitrarily far from equilibrium is why this formulation is also known as Near-Equilibrium Thermodynamics.

At any rate, having now identified the realm of applicability of the theory, we can move to its mathematical expression, starting with the laws of conservation of energy and mass in local form, to eventually arrive at a balance equation for the entropy density.

\subsection{Conservation of Mass}

Let us consider a system consisting on $\mathrm{n}$ components among which $r$ chemical reactions are possible. The rate of change of the mass of component $k$ within a given volume $V$ is $[4]$ :

$$
\frac{d}{d t} \int_{V} \rho_{k}(x, y, z) \mathrm{d} V=\int_{V} \frac{\partial \rho_{k}}{\partial t} \mathrm{~d} V
$$

Where $\rho_{k}$ is the density of component $k$. This quantity is equal to the sum of the material flow of component $k$ into the volume $V$ through its surface $\Sigma$ and the total production of $k$ in chemical reactions occurring inside $V$ :

$$
\int_{V} \frac{\partial \rho_{k}}{\partial t} \mathrm{~d} V=-\oint_{\Sigma} \rho_{k} \vec{v}_{k} \cdot \mathrm{d} \vec{\Sigma}+\sum_{j=1}^{r} \int_{V} \nu_{k j} J_{j} \mathrm{~d} V
$$

Where $\mathrm{d} \vec{\Sigma}$ is a vector with magnitude $\mathrm{d} \Sigma$ normal to the surface element and oriented toward the exterior. The vector $\vec{v}_{k}$ is the velocity of component $k$ 's centre of mass and $\nu_{k j} J_{j}$ the production of $k$ per unit volume in the $j^{\text {th }}$ chemical reaction. The quantity $\nu_{k j}$ divided by the molecular mass $M_{k}$ of component $k$ is proportional to the stoichiometric coefficient with which $k$ appears in the chemical reaction $j$. The coefficients $\nu_{k j}$ are counted positive if they appear as products of the reaction and negative if they appear as reactants. The quantity $J_{j}$ is called the chemical reaction rate of reaction $j$, and its dimensions are of a mass per unit volume and unit time.

\footnotetext{
${ }^{2}$ At most the discontinuities can occur over subsets of $\Omega$ of zero measure.
} 
Applying Gauss' theorem to the surface integral in (1.4), since the relation is valid for an arbitrary volume $V$, we obtain:

$$
\frac{\partial \rho_{k}}{\partial t}=-\nabla \cdot\left(\rho_{k} \vec{v}_{k}\right)+\sum_{j=1}^{r} \nu_{k j} J_{j} \quad, \quad k=1,2, \ldots n
$$

Which has the form of what is known as a balance equation, since it relates the rate of change of a quantity to the divergence of a flux and to a source term. In this case the flux is the amount of mass of chemical species $k$ traversing the boundary of the volume per unit time and unit area, while the source term is evidently the total production of component $k$ within the volume per unit time, or $\sum_{j=1}^{r} \nu_{k j} J_{j}$. Since mass is conserved in each separate chemical reaction we have:

$$
\sum_{k=1}^{n} \nu_{k j}=0 \quad, \quad j=1,2, \ldots, r
$$

Given this, by summing the equation over all components $k$ one obtains the law of conservation of mass for a system of $n$ chemical species undergoing $r$ different chemical reactions, such that a well-defined density and velocity field exist locally throughout:

$$
\frac{\partial \rho}{\partial t}=-\nabla \cdot(\rho \vec{v})
$$

Where $\rho$ is the total density:

$$
\rho=\sum_{k=1}^{n} \rho_{k}
$$

And $\vec{v}$ is the centre of mass velocity of the mixture:

$$
\vec{v}=\sum_{k=1}^{n} \frac{\rho_{k}}{\rho} \vec{v}_{k}
$$

The continuity equation (1.7) expresses the fact that the total mass in the system is conserved. That is, the total mass in any volume element, as defined by (1.8), can only change if matter flows into (or out of) the volume.

We can rewrite the mass equations in an more succinct way by use of the total time derivative (i.e. the material derivative):

$$
\frac{\mathrm{d}}{\mathrm{d} t}=\frac{\partial}{\partial t}+\vec{v} \cdot \nabla
$$


Where the term $\vec{v} \cdot \nabla$ is known as the advection term, and by introducing the diffusion flow $\vec{J}_{k}$ of substance $k$ with respect to the centre of mass motion of the volume element:

$$
\overrightarrow{J_{k}}=\rho_{k}\left(\vec{v}_{k}-\vec{v}\right)
$$

Employing both (1.10) and (1.11) the balance equation (1.5) thus becomes:

$$
\frac{\mathrm{d} \rho_{k}}{\mathrm{~d} t}=-\rho_{k} \nabla \cdot \vec{v}-\nabla \cdot \vec{J}_{k}+\sum_{j=1}^{r} \nu_{k j} J_{j} \quad, \quad k=1,2, \ldots, n
$$

And equation (1.7):

$$
\frac{\mathrm{d} \rho}{\mathrm{d} t}=-\rho \nabla \cdot \vec{v}
$$

Or in terms of the total specific volume $v=\rho^{-1}$ and by use of the chain rule as:

$$
\rho \frac{\mathrm{d} v}{\mathrm{~d} t}=\nabla \cdot \vec{v}
$$

If we define the mass fractions $c_{k}=\rho_{k} / \rho$ and employ them in equation (1.12) along with (1.13), we turn the balance equation, as promised, into its tersest form:

$$
\rho \frac{\mathrm{d} c_{k}}{\mathrm{~d} t}=-\nabla \cdot \vec{J}_{k}+\sum_{j=1}^{r} \nu_{k j} J_{j} \quad, \quad k=1,2, \ldots, n
$$

As a final remark in this section on mass conservation, we seek to derive a result that shall prove invaluable in all sections to follow. As a consequence of equation (1.13) and the total derivative operator identity (1.10), for an arbitrary local quantity $\gamma(\vec{r}, t)$ characterising in some way the local state of the system at the point $(\vec{r}, t)$, the following always holds:

$$
\rho \frac{\mathrm{d} \gamma}{\mathrm{d} t}=\frac{\partial(\gamma \rho)}{\partial t}+\nabla \cdot(\gamma \rho \vec{v})
$$

In fact, from the product rule and the total derivative one has:

$$
\begin{aligned}
& \text { (i) } \frac{\mathrm{d}(\gamma \rho)}{\mathrm{d} t}=\gamma \frac{\mathrm{d} \rho}{\mathrm{d} t}+\rho \frac{\mathrm{d} \gamma}{\mathrm{d} t} \\
& \text { (ii) } \frac{\mathrm{d}(\gamma \rho)}{\mathrm{d} t}=\nabla(\gamma \rho) \cdot \vec{v}+\frac{\partial(\gamma \rho)}{\partial t} \Rightarrow
\end{aligned}
$$




$$
\begin{aligned}
\gamma \frac{\mathrm{d} \rho}{\mathrm{d} t}+\rho \frac{\mathrm{d} \gamma}{\mathrm{d} t} & =\nabla \cdot(\gamma \rho \vec{v})-\gamma \rho \nabla \cdot \vec{v}+\frac{\partial(\gamma \rho)}{\partial t} \Rightarrow \\
\gamma \frac{\mathrm{d} \rho}{\mathrm{d} t}+\rho \frac{\mathrm{d} \gamma}{\mathrm{d} t} & =\nabla \cdot(\gamma \rho \vec{v})+\gamma \frac{\mathrm{d} \rho}{\mathrm{d} t}+\frac{\partial(\gamma \rho)}{\partial t} \Rightarrow \\
\rho \frac{\mathrm{d} \gamma}{\mathrm{d} t} & =\nabla \cdot(\gamma \rho \vec{v})+\frac{\partial(\gamma \rho)}{\partial t} \quad \square .
\end{aligned}
$$

\subsection{Equations of Motion}

To arrive at an expression for the law of conservation of energy we naturally begin with the equations of motion of the system's centre of mass. In complete analogy to the continuum model of hydrodynamics, we divide in two the forces acting on our volume element $\mathrm{d} V$, one due to the pressure tensor $P$ (of rank two) acting on the surface of the volume $\Sigma$, and another due to the forces per unit mass $\vec{F}_{k}$ exerted on the chemical component $k$.

The equations of motion for the centre of mass is thus written as:

$$
\rho \frac{\mathrm{d} v_{i}}{\mathrm{~d} t}=-\sum_{j=1}^{3} \frac{\partial P_{j i}}{\partial x_{j}}+\sum_{k=1}^{n} \rho_{k} F_{k i} \quad, \quad i=1,2,3
$$

Or in the more concise vector notation:

$$
\rho \frac{\mathrm{d} \vec{v}}{\mathrm{~d} t}=-\nabla \cdot P+\sum_{k} \rho_{k} \vec{F}_{k}
$$

From a microscopic point of view, the pressure tensor $P$ is regarded as involving the short-range interactions between particles, whereas $\vec{F}_{k}$ contains the external forces as well as long-range interactions in the system [4]. We restrict our discussion of forces $\vec{F}_{k}$ to conservative ones independent of time, i.e. that can be expressed as the gradient of some potential energy $\psi_{k}$ :

$$
\vec{F}_{k}=-\nabla \psi_{k} \quad, \quad \frac{\partial \psi_{k}}{\partial t}=0
$$


It is instructive to note that we can rewrite the equation of motion (1.18) with the help of (1.16) derived at the end of the last section and obtain a balance equation for the momentum density $\rho \vec{v}$ :

$$
\frac{\partial \rho \vec{v}}{\partial t}=-\nabla \cdot(\rho \vec{v} \vec{v}+P)+\sum_{k} \rho_{k} \vec{F}_{k}
$$

Where $\vec{v} \vec{v}=v_{i} v_{j}(i, j=1,2,3)$ stands for the ordered dyadic product of $\vec{v}$ with itself, i.e.a 3-dimensional square matrix obtained from the tensor product of the velocity with itself. Now it is clear that one can interpret the term $\rho \vec{v} \vec{v}+P$ as a momentum flow due to $P$ with a convective part $^{3} \rho \vec{v} \vec{v}$, while the quantity $\sum_{k} \rho_{k} \vec{F}_{k}$ is a source term of momentum density. Now we seek to derive a balance equation for the kinetic energy of the centre of mass motion. By taking the equations of motion in component form (1.17) and contracting both sides with the centre of mass velocity $v_{i}$ one has:

$$
\begin{gathered}
\rho \sum_{i=1}^{3} v^{i} \frac{\mathrm{d} v_{i}}{\mathrm{~d} t}=-\sum_{i, j=1}^{3} v^{i} \frac{\partial P_{j i}}{\partial x_{j}}+\sum_{k=1}^{n} \sum_{i=1}^{3} \rho_{k} v^{i} F_{k i} \\
\rho \frac{\mathrm{d} \frac{1}{2} v^{2}}{\mathrm{~d} t}=-\sum_{i, j=1}^{3} \frac{\partial\left(v^{i} P_{j i}\right)}{\partial x_{j}}+\sum_{i, j=1}^{3} P_{j i} \frac{\partial v^{i}}{\partial x_{j}}+\sum_{k=1}^{n} \sum_{i=1}^{3} \rho_{k} v^{i} F_{k i} \Rightarrow \\
\rho \frac{\mathrm{d} \frac{1}{2} v^{2}}{\mathrm{~d} t}=-\nabla \cdot(P \vec{v})+P: \nabla \vec{v}+\sum_{k} \rho_{k} \vec{F}_{k} \cdot \vec{v}
\end{gathered}
$$

Where the symbol : stands for the contraction of $P$ and $\nabla \vec{v}$ over all indices:

$$
P: \nabla \vec{v} \equiv \sum_{i, j=1}^{3} P_{j i} \frac{\partial v^{i}}{\partial x_{j}}
$$

Now, employing yet again the identity (1.16) we can obtain from (1.21) a balance equation for the kinetic energy density $\frac{1}{2} \rho v^{2}$ :

$$
\frac{\partial \frac{1}{2} \rho v^{2}}{\partial t}=-\nabla \cdot\left(\frac{1}{2} \rho v^{2} \vec{v}+P \vec{v}\right)+P: \nabla \vec{v}+\sum_{k} \rho_{k} \vec{F}_{k} \cdot \vec{v}
$$

\footnotetext{
${ }^{3}$ Convective terms in divergences of flux quantities occur throughout the theory, since the rate of change of a given quantity can also be due to the centre of mass' own macroscopic flow; for example in the case of the momentum density, the centre of mass of the volume $\mathrm{d} V$ may travel to regions of $\Omega$ where the momentum density takes a different value to that of the volume, leading to net exchange of that quantity, whether favourable or not.
} 
Having now derived a balance equation for the kinetic energy of the centre of mass motion, we want to add the potential energy density contribution and obtain a balance equation for the total centre of mass energy. Therefore, defining the total potential energy density as $\rho \psi=\sum_{k} \rho_{k} \psi_{k}$, employing (1.5), (1.11) along the way and remembering that for each $k=1,2, \ldots, n \psi_{k}$ doesn't depend on time explicitly, we obtain:

$$
\begin{gathered}
\frac{\partial(\rho \psi)}{\partial t}=\sum_{k=1}^{n} \psi_{k} \frac{\partial \rho_{k}}{\partial t}+\sum_{k=1}^{n} \rho_{k} \frac{\partial \psi_{k}}{\partial t}=\sum_{k=1}^{n} \psi_{k} \frac{\partial \rho_{k}}{\partial t} \\
\frac{\partial \rho_{k}}{\partial t}=-\nabla \cdot\left(\rho_{k} \vec{v}_{k}\right)+\sum_{j=1}^{r} \nu_{k j} J_{j} \quad, \quad(k=1,2, \ldots n) \Rightarrow \\
\frac{\partial(\rho \psi)}{\partial t}=-\sum_{k=1}^{n} \nabla \cdot\left(\rho_{k} \vec{v}_{k}\right) \psi_{k}+\sum_{k=1}^{n} \sum_{j=1}^{r} \nu_{k j} \psi_{k} J_{j} \Rightarrow \\
=-\sum_{k=1}^{n} \nabla \cdot\left(\vec{J}_{k}+\rho_{k} \vec{v}\right) \psi_{k}+\sum_{k=1}^{n} \sum_{j=1}^{r} \nu_{k j} \psi_{k} J_{j} \Rightarrow \\
=-\sum_{k=1}^{n} \nabla \cdot\left(\overrightarrow{J_{k}} \psi_{k}+\rho_{k} \psi_{k} \vec{v}\right)+\sum_{k=1}^{n}\left(\vec{J}_{k}+\rho_{k} \vec{v}_{k}\right) \cdot \nabla \psi_{k}+\sum_{k=1}^{n} \sum_{j=1}^{r} \nu_{k j} \psi_{k} J_{j} \Rightarrow \\
\frac{\partial(\rho \psi)}{\partial t}=-\nabla \cdot\left(\rho \psi \vec{v}+\sum_{k=1}^{n} \psi_{k} \overrightarrow{J_{k}}\right)-\sum_{k=1}^{n} \rho_{k} \vec{F}_{k} \cdot \vec{v}-\sum_{k=1}^{n} \vec{J}_{k} \cdot \vec{F}_{k}+\sum_{k=1}^{n} \sum_{j=1}^{r} \nu_{k j} \psi_{k} J_{j}
\end{gathered}
$$

The last term vanishes if the potential energy is conserved in a chemical reaction:

$$
\sum_{k} \psi_{k} \nu_{k j}=0 \quad, \quad j=1,2, \ldots, r
$$

If the property of the particles (i.e. electric charge in an electric field or mass in gravitational field) responsible for the interaction with a field of force is conserved, then this happens to be the case [4]. Supposing this is the regime of applicability then equation (1.23) reduces to:

$$
\frac{\partial(\rho \psi)}{\partial t}=-\nabla \cdot\left(\rho \psi \vec{v}+\sum_{k=1}^{n} \psi_{k} \overrightarrow{J_{k}}\right)-\sum_{k=1}^{n} \rho_{k} \vec{F}_{k} \cdot \vec{v}-\sum_{k=1}^{n} \vec{J}_{k} \cdot \vec{F}_{k}
$$


Summing together the rates of change for the kinetic energy (1.22) and potential energy density (1.25) we finally have:

$$
\frac{\partial \rho\left(\psi+\frac{1}{2} v^{2}\right)}{\partial t}=-\nabla \cdot\left[\rho \vec{v}\left(\frac{1}{2} v^{2}+\psi\right)+P \vec{v}+\sum_{k=1}^{n} \psi_{k} \overrightarrow{J_{k}}\right]+P: \nabla \vec{v}-\sum_{k=1}^{n} \vec{J}_{k} \cdot \vec{F}_{k}
$$

Where it becomes clear that this quantity is not conserved after all, since a source term appears on the right. This immediately tells us that for the law of conservation of energy to hold there must be a term missing in this expression for the total specific energy; it cannot simply be the sum of the specific potential energy and the specific kinetic energy $\frac{1}{2} v^{2}+\psi$. We identify this missing contribution as being the specific internal energy $u$, which we can in fact define as the difference between the total specific energy $e$, which must be conserved, and the specific centre of mass energy $\frac{1}{2} v^{2}+\psi$ :

$$
e=\frac{1}{2} v^{2}+\psi+u
$$

In fact, the definition we gave of centre of mass energy as the sum of kinetic and potential terms doesn't include the microscopic contributions to the total energy of the system given by heat and interactions between particles occuring internally.

In fact, along with the macroscopic centre of mass kinetic energy described by $\vec{v}$ and the external forces $\vec{F}_{k}$, uncorrelated thermal agitations of the single particles and short-range interactions are clearly present (i.e intermolecular forces: covalent and ionic bonds, induced dipole forces and van der Waals forces). Therefore $u$ is the part of the specific total energy which encodes within it all these contributions and thus plays the same role here as in equilibrium thermodynamics, where $U$ neither contains a term of kinetic energy of the centre of mass ${ }^{4}$ nor any potential energy contributions from external force fields acting on the system [2].

\subsection{Conservation of Energy}

The principle of conservation of energy states that the total energy contained within an arbitrary volume $V$ can only change if energy flows into or out of the volume element through its boundary $\partial V=\Sigma$. Since energy cannot be produced nor erased, it obeys a balance equation without source terms. Therefore if $\vec{J}_{e}$ represents the energy flux per unit area and unit time flowing through the boundary of $V$, the law of energy conservation can be stated as:

\footnotetext{
${ }^{4} U$ is defined in the reference frame where the centre of mass is stationary.
} 


$$
\frac{\mathrm{d}}{\mathrm{d} t} \int_{V} \rho e \mathrm{~d} V=\int_{V} \frac{\partial \rho e}{\partial t} \mathrm{~d} V=-\oint_{\Sigma} \overrightarrow{J_{e}} \cdot \mathrm{d} \vec{\Sigma}
$$

Where once again $e$ is the energy per unit mass. Given the arbitrariness of the volume $V$, Gauss' theorem allows us to obtain a differential or local expression of the energy conservation law:

$$
\frac{\partial \rho e}{\partial t}=-\nabla \cdot \overrightarrow{J_{e}}
$$

The total energy flux $\vec{J}_{e}$ includes a convective term $\rho e \vec{v}$, an energy flux $P \cdot \vec{v}$ due to the mechanical work performed on the volume element, a potential energy flux $\sum_{k} \psi_{k} \overrightarrow{J_{k}}$ due to the diffusion of the different components in the force field, and a "heat flow" $\vec{J}_{q}$ $[4]$ :

$$
\overrightarrow{J_{e}}=\rho e \vec{v}+P \cdot \vec{v}+\sum_{k=1}^{n} \psi_{k} \vec{J}_{k}+\vec{J}_{q}
$$

This expression may be considered as defining the "heat flow" $\vec{J}_{q}$. Consistently with our usual notion of heat (far from phase transitions), the term $\vec{J}_{q}$ arises from the generalized collision processes happening near the boundary $\Sigma$ of our volume $V$, however the heat flux also contains an additional term, given from the material flows of particles entering or leaving the volume and carrying each a variable amount of specific "enthalpy" [4]. If we now combine equations (1.26), (1.29), (1.27) and (1.30) we obtain the balance equation for the specific internal energy $u$ :

$$
\begin{gathered}
\left\{\frac{\partial \rho u}{\partial t}=\frac{\partial \rho e}{\partial t}-\frac{\partial \rho\left(\psi+\frac{1}{2} v^{2}\right)}{\partial t} \wedge \frac{\partial \rho e}{\partial t}=-\nabla \cdot \vec{J}_{e} \wedge\right. \\
\left\{\frac{\partial \rho\left(\psi+\frac{1}{2} v^{2}\right)}{\partial t}=-\nabla \cdot\left[\rho \vec{v}\left(\frac{1}{2} v^{2}+\psi\right)+P \vec{v}+\sum_{k=1}^{n} \psi_{k} \vec{J}_{k}\right]+P: \nabla \vec{v}-\sum_{k=1}^{n} \vec{J}_{k} \cdot \vec{F}_{k}\right. \\
\Rightarrow \frac{\partial \rho u}{\partial t}=-\nabla \cdot \overrightarrow{J_{e}}+\nabla \cdot\left[\rho \vec{v}\left(\frac{1}{2} v^{2}+\psi\right)+P \vec{v}+\sum_{k=1}^{n} \psi_{k} \vec{J}_{k}\right]-P: \nabla \vec{v}+\sum_{k=1}^{n} \vec{J}_{k} \cdot \vec{F}_{k} \Rightarrow \\
\frac{\partial \rho u}{\partial t}=-\nabla \cdot\left(\rho e \vec{v}+P \cdot \vec{v}+\sum_{k=1}^{n} \psi_{k} \vec{J}_{k}+\overrightarrow{J_{q}}\right)+\nabla \cdot\left[\rho \vec{v}\left(\frac{1}{2} v^{2}+\psi\right)+P \vec{v}+\sum_{k=1}^{n} \psi_{k} \vec{J}_{k}\right] \\
-P: \nabla \vec{v}+\sum_{k=1}^{n} \vec{J}_{k} \cdot \vec{F}_{k}
\end{gathered}
$$




$$
\begin{gathered}
\Rightarrow \frac{\partial \rho u}{\partial t}=-\nabla \cdot\left[\rho \vec{v}\left(e-\frac{1}{2} v^{2}-\psi\right)\right]-\nabla \cdot \vec{J}_{q}-P: \nabla \vec{v}+\sum_{k=1}^{n} \vec{J}_{k} \cdot \vec{F}_{k} \\
\Rightarrow \frac{\partial \rho u}{\partial t}=-\nabla \cdot\left(\rho u \vec{v}+\vec{J}_{q}\right)-P: \nabla \vec{v}+\sum_{k=1}^{n} \vec{J}_{k} \cdot \vec{F}_{k}
\end{gathered}
$$

It is apparent that the specific internal energy $u$ cannot be a conserved quantity in general, since a source term appears on the right-hand side of its balance equation.

The source term is equal but of opposite sign to the source term appearing in the centre of mass energy balance equation (1.26). This fact confirms what was speculated in the previous section, that the internal energy can change due to heat flow (generalised collisions occuring on the boundary $\Sigma=\partial V$ of $\mathrm{V}$ leading to energy exchange), short-range interactions exerting work on the volume element $P: \nabla \vec{v}$, work exerted on the individual chemical species by the specific long-range external forces $\vec{J}_{k} \cdot \vec{F}_{k}$ (which disappear if the volume is closed) and lastly, gain or loss of energy due to the ever-present convective term $\rho\left(e-\frac{1}{2} v^{2}-\psi\right) \vec{v}$.

\subsection{Local Expression of the First Law}

We are now close to the primary goal of this chapter: the derivation of the first law of thermodynamics in differential form for quasi-equilibrium systems (i.e. locally in equilibrium). Assuming we can split the pressure tensor into a scalar hydrostatic part $p$ and a non-scalar tensor $\Pi^{5}$ :

$$
P=p I+\Pi
$$

Where $I$ is the $3 \times 3$ identity matrix. Employing relation (1.16) yet again, we can recast the balance equation (1.31):

$$
\begin{aligned}
& \rho \frac{\mathrm{d} u}{\mathrm{~d} t}=-\nabla \cdot \vec{J}_{q}-p \nabla \cdot \vec{v}-\Pi: \nabla \vec{v}+\sum_{k=1}^{n} \vec{J}_{k} \cdot \vec{F}_{k} \\
& \rho \frac{\mathrm{d} u}{\mathrm{~d} t}=-\rho \frac{\mathrm{d} q}{\mathrm{~d} t}-p \nabla \cdot \vec{v}-\Pi: \nabla \vec{v}+\sum_{k=1}^{n} \vec{J}_{k} \cdot \vec{F}_{k}
\end{aligned}
$$

\footnotetext{
${ }^{5}$ Assuming that a hydrostatic component of the pressure can be isolated means we choose to limit the discussion to non-elastic fluids [4]. Naturally, in elastic mediums the pressure tensor is replaced by the elastic stress tensor and the simplifaction that follows is not applicable.
} 
Where we have used the fact that when the identity matrix contracts with the gradient $\nabla$ it maps it into the divergence $\nabla \cdot$, in fact (using Einstein's summation convention):

$$
I: \nabla \vec{v}=\delta_{i}^{j} \partial_{j} v^{i}=\partial_{i} v^{i}=\nabla \cdot \vec{v}
$$

And where we have defined the infinitesimal "heat" $\mathrm{d} q$ added per unit mass as:

$$
\rho \frac{\mathrm{d} q}{\mathrm{~d} t}=-\nabla \cdot \vec{J}_{q}
$$

Remembering (1.14) for the specific volume $v$ 's rate of change, we can now finally express the "first law of linear irreversible thermodynamics" for non-elastic media in conservative force fields in differential form:

$$
\frac{\mathrm{d} u}{\mathrm{~d} t}=\frac{\mathrm{d} q}{\mathrm{~d} t}-p \frac{\mathrm{d} v}{\mathrm{~d} t}-v \Pi: \nabla \vec{v}+v \sum_{k=1}^{n} \vec{J}_{k} \cdot \vec{F}_{k}
$$




\section{Chapter 2}

\section{The Second Law of Thermodynamics}

\subsection{Local Expression of the Second Law}

According to the principles of equilibrium thermodynamics, one can introduce for any system in equilibrium a state function $\mathrm{S}$ known as the entropy. In $1865 \mathrm{R}$. Clausius discovered that while the amount of heat $\delta Q$ exchanged by the system with the surroundings as it undergoes an infinitesimal and reversible transformation is not an exact differential ( $Q$ depends on the particular trajectory in state space), when divided by the absolute temperature $T$ it becomes one [1]. In other words, the line integral of this quantity along a reversible cycle $\gamma$ is identically zero:

$$
\oint_{\gamma} \frac{\delta Q}{T}=0
$$

And that therefore the line integral along any curve $\Gamma$ will be function only of its endpoints $\mathrm{A}$ and $\mathrm{B}$, thus defining a state function called the entropy S:

$$
\int_{B}^{A} \frac{\delta Q}{T}=S(A)-S(B)
$$

Suppose now that we can always write the differential of the entropy as the sum of two terms:

$$
\mathrm{d} S=\mathrm{d} S_{i}+\mathrm{d} S_{e}
$$

Where $\mathrm{d} S_{e}$ is the entropy supplied by the surroundings and $\mathrm{d} S_{i}$ the entropy produced within the system [4]. The second law of thermodynamics states that $\mathrm{d} S_{i}$ must be zero 
for reversible (or quasi-static) transformations of the system, and strictly positive for irreversible ones [5]:

$$
\mathrm{d} S_{i} \geq 0
$$

The entropy supplied $\mathrm{d} S_{e}$ however can be negative, positive or null, depending on the particular interaction between the system and the environment [4]. For an adiabatically insulated system, $\delta Q$ will be zero by definition and therefore so will the term $\mathrm{d} S_{e}$, and we'll have another well-known restatement of the second law:

$$
\mathrm{d} S \geq 0 \quad \text { for adiabatically closed systems }
$$

For closed systems, we have according to the theorem of Clausius-Carnot [1]:

$$
\begin{gathered}
\mathrm{d} S_{e}=\frac{\delta Q}{T} \quad \text { closed } \\
\mathrm{d} S \geq \frac{\delta Q}{T} \quad \text { closed }
\end{gathered}
$$

It is important to note that clearly for open systems (2.6) and (2.7) cease to be valid, while (2.3) and (2.4) are of a completely general nature. In complete analogy with the preceding chapters, we seek to restate (2.3) and (2.4) in integral form, so as to be more suitable for systems described by extensive state variable that are differentiable functions of space and time:

$$
\begin{gathered}
S=\int_{V} \rho s \mathrm{~d} V \\
\frac{\mathrm{d} S_{e}}{\mathrm{~d} t}=-\oint_{\Sigma} \vec{J}_{s, t o t} \cdot \mathrm{d} \vec{\Sigma} \\
\frac{\mathrm{d} S_{i}}{\mathrm{~d} t}=\int_{V} \sigma \mathrm{d} V
\end{gathered}
$$

Where $s$ is the entropy per unit mass, $\vec{J}_{s, t o t}$ the total entropy flux flowing through the closed boundary $\Sigma$ of $V$, and $\sigma$ the entropy production within $V$ occurring per unit volume and unit time i.e the entropy density production rate or entropy source strength [4]. With the help of Gauss' theorem, the arbitrariness of the volume $V$, and these new definitions, we can rewrite (2.3) and (2.4) in local form like so:

$$
\int_{V} \frac{\partial \rho s}{\partial t} \mathrm{~d} V=\frac{\mathrm{d} S}{\mathrm{~d} t}=\frac{\mathrm{d} S_{i}}{\mathrm{~d} t}+\frac{\mathrm{d} S_{e}}{\mathrm{~d} t}=-\int_{V} \nabla \cdot \vec{J}_{s, t o t} \mathrm{~d} V+\int_{V} \sigma \mathrm{d} V \Rightarrow
$$




$$
\begin{gathered}
\int_{V}\left(\frac{\partial \rho s}{\partial t}+\nabla \cdot \vec{J}_{s, t o t}-\sigma\right) \mathrm{d} V=0 \Rightarrow \\
\frac{\partial \rho s}{\partial t}=-\nabla \cdot \vec{J}_{s, t o t}+\sigma \\
\sigma \geq 0
\end{gathered}
$$

Equation (2.11) is formally a balance equation for the rate of change of entropy density within $V$, with a source term $\sigma$ satisfying the local equivalent of (2.4). Invoking once more relation (1.16) we can rewrite (2.11) in the following way:

$$
\rho \frac{\mathrm{d} s}{\mathrm{~d} t}=-\nabla \cdot \overrightarrow{J_{s}}+\sigma
$$

Where as usual we see the appearance of a convective term:

$$
\overrightarrow{J_{s}}=\vec{J}_{s, t o t}-\rho s \vec{v}
$$

\subsection{Entropy Flux Density and Production Rate}

Putting everything we have learned in the previous chapters together, we will arrive at an explicit expression for the entropy balance equation, since at the moment, the entropy flux $\vec{J}_{s}$ and production rate $\sigma$ lack a precise expression in terms of the relevant physical quantities we have introduced thus far. Assuming the system is locally in equilibrium, and that we have written the entropy per unit mass as a function of the internal energy density $u$, the specific volume $v=\rho^{-1}$ and the mass fractions $c_{k}$ of the $k$ chemical components:

$$
s=s\left(u, v, c_{k}\right)
$$

At equilibrium, the total differential of $s$ is written as the Gibbs relation [4]:

$$
T \mathrm{~d} s=\mathrm{d} u+p \mathrm{~d} v-\sum_{k=1}^{n} \mu_{k} \mathrm{~d} c_{k}
$$

Assuming that (2.16) remains valid along the centre of mass motion of the volume element, we can rewrite the expression in terms of the material derivatives of the entropy density and the parameters it's a function of $\left(u, v, \mu_{k}\right)$ : 


$$
T \frac{\mathrm{d} s}{\mathrm{~d} t}=\frac{\mathrm{d} u}{\mathrm{~d} t}+p \frac{\mathrm{d} v}{\mathrm{~d} t}-\sum_{k=1}^{n} \mu_{k} \frac{\mathrm{d} c_{k}}{\mathrm{~d} t}
$$

The situations in which this assumption holds can only be identified a posteriori, in virtue of the validity of the predictions derived from it [4]. In some special cases the validity of (2.17) can be shown to be valid a priori if the system considered is near equilibrium. Nearness to equilibrium is rigorously defined in terms of the deviations that thermodynamic quantities have from their equilibrium values. In some special cases, the orders of magnitude of these fluctuations are computable and it is in these cases that we can justify a priori the validity of (2.17) [4]. At any rate, we can now find the explicit statement of the entropy balance equation by inserting the first law in local form (1.36) and the expression for the mass fractions rates of change (1.15) into (2.17):

$$
\rho \frac{\mathrm{d} c_{k}}{\mathrm{~d} t}=-\nabla \cdot \vec{J}_{k}+\sum_{j=1}^{r} \nu_{k j} J_{j}
$$

$$
\frac{\mathrm{d} u}{\mathrm{~d} t}=\frac{\mathrm{d} q}{\mathrm{~d} t}-p \frac{\mathrm{d} v}{\mathrm{~d} t}-v \Pi: \nabla \vec{v}+v \sum_{k=1}^{n} \vec{J}_{k} \cdot \vec{F}_{k}
$$

$$
T \frac{\mathrm{d} s}{\mathrm{~d} t}=\frac{\mathrm{d} u}{\mathrm{~d} t}+p \frac{\mathrm{d} v}{\mathrm{~d} t}-\sum_{k=1}^{n} \mu_{k} \frac{\mathrm{d} c_{k}}{\mathrm{~d} t} \quad \Rightarrow
$$

$$
\begin{aligned}
& T \frac{\mathrm{d} s}{\mathrm{~d} t}= \frac{\mathrm{d} q}{\mathrm{~d} t}-p \frac{\mathrm{d} v}{\mathrm{~d} t}+p \frac{\mathrm{d} v}{\mathrm{~d} t}-\sum_{k=1}^{n} \mu_{k} \frac{\mathrm{d} c_{k}}{\mathrm{~d} t}-v \Pi: \nabla \vec{v}+v \sum_{k=1}^{n} \vec{J}_{k} \cdot \vec{F}_{k} \Rightarrow \\
& T \frac{\mathrm{d} s}{\mathrm{~d} t}=-v \nabla \cdot \overrightarrow{J_{q}}-\sum_{k=1}^{n} \mu_{k} \frac{\mathrm{d} c_{k}}{\mathrm{~d} t}-v \Pi: \nabla \vec{v}+v \sum_{k=1}^{n} \vec{J}_{k} \cdot \vec{F}_{k} \Rightarrow \\
& T \frac{\mathrm{d} s}{\mathrm{~d} t}=-v \nabla \cdot \vec{J}_{q}-\sum_{k=1}^{n} v \mu_{k}\left(\nabla \cdot \vec{J}_{k}+\sum_{j=1}^{r} \nu_{k j} J_{j}\right)-v \Pi: \nabla \vec{v}+v \sum_{k=1}^{n} \vec{J}_{k} \cdot \vec{F}_{k} \Rightarrow \\
& \rho T \frac{\mathrm{d} s}{\mathrm{~d} t}=-\nabla \cdot \vec{J}_{q}-\Pi: \nabla \vec{v}+\sum_{k=1}^{n} \vec{J}_{k} \cdot \vec{F}_{k}+\sum_{k=1}^{n} \mu_{k} \nabla \cdot \vec{J}_{k}-\sum_{j=1}^{r} A_{j} J_{j}
\end{aligned}
$$

Where we have defined the chemical affinities $A_{j}$ of the reactions $j=1,2, \ldots, r$ as: 


$$
A_{j}=\sum_{k=1}^{n} \nu_{k j} \mu_{k}
$$

Now recasting (2.18) in the form of a balance equation of the same form as (2.13):

$$
\begin{aligned}
& \rho \frac{\mathrm{d} s}{\mathrm{~d} t}=-\nabla \cdot\left(\frac{\overrightarrow{J_{q}}-\sum_{k} \mu_{k} \overrightarrow{J_{k}}}{T}\right)-\frac{1}{T^{2}} \vec{J}_{q} \cdot \nabla T-\frac{1}{T} \sum_{k=1}^{n} \vec{J}_{k} \cdot\left[T \nabla\left(\frac{\mu_{k}}{T}\right)-\vec{F}_{k}\right] \\
& -\frac{1}{T} \Pi: \nabla \vec{v}-\frac{1}{T} \sum_{j=1}^{r} A_{j} J_{j}
\end{aligned}
$$

Lastly, comparing coefficients with (2.13) yields an explicit expression for the entropy flux density $\overrightarrow{J_{s}}$ and the production rate $\sigma$ in terms other underlying quantities:

$$
\begin{gathered}
\vec{J}_{s}=\frac{1}{T}\left(\vec{J}_{q}-\sum_{k=1}^{n} \mu_{k} \vec{J}_{k}\right) \\
\sigma=-\frac{1}{T^{2}} \vec{J}_{q} \cdot \nabla T-\frac{1}{T} \sum_{k=1}^{n} \vec{J}_{k} \cdot\left[T \nabla\left(\frac{\mu_{k}}{T}\right)-\vec{F}_{k}\right]-\frac{1}{T} \Pi: \nabla \vec{v}-\frac{1}{T} \sum_{j=1}^{r} A_{j} J_{j}
\end{gathered}
$$

We can see that entropy is produced and exchanged due to gradients of thermodynamic parameters (temperature, chemical potential, velocity), divergences of matter and energy fluxes $\overrightarrow{J_{k}}, \overrightarrow{J_{q}}$, work per unit time done on the system by external forces, and changes in chemical potential per unit time due to ongoing chemical reactions.

Although it seems arbitrary how this equation was recast into the divergence of a flux and a source term, the requirements that the two parts must satisfy determine this separation uniquely [4].

In fact, the entropy production rate $\sigma$ needs to be invariant under Galilean transformations, for the notions of reversibility and irreversibility cannot be relative concepts depending on our frame of reference. In addition, $\sigma$ should equal zero for systems which are in equilibrium. The proof of both these statements, which happen to hold for $\sigma$, can be found in the Appendix (A.1).

Finally, summing (2.20) and (2.21) together and integrating over the volume $\mathrm{V}$ of a closed system, keeping in mind the inequality $\sigma \geq 0$, we recover the local equilibrium equivalent of the Carnot-Clausius theorem (2.7), namely: 


$$
\begin{aligned}
\int_{V} \rho \frac{\mathrm{d} s}{\mathrm{~d} t} \mathrm{~d} V & =\int_{V}-\nabla \cdot \overrightarrow{J_{s}} \mathrm{~d} V+\int_{V} \sigma \mathrm{d} V \\
\frac{\mathrm{d} S}{\mathrm{~d} t} & \geq-\int_{V} \nabla \cdot\left(\frac{\overrightarrow{J_{q}}-\sum_{k} \mu_{k} \overrightarrow{J_{k}}}{T}\right) \mathrm{d} V \\
\frac{\mathrm{d} S}{\mathrm{~d} t} & \geq \oint_{\Sigma} \frac{\overrightarrow{J_{q}}}{T} \cdot \mathrm{d} \vec{\Sigma}+\oint_{\Sigma} \frac{\sum_{k} \mu_{k} \overrightarrow{J_{k}}}{T} \cdot \mathrm{d} \vec{\Sigma} \\
\frac{\mathrm{d} S}{\mathrm{~d} t} & \geq \oint_{\Sigma} \frac{\overrightarrow{J_{q}}}{T} \cdot \mathrm{d} \vec{\Sigma}
\end{aligned}
$$

Where we used the fact that if our system, contained in the volume $V$, is closed, then by definition $\overrightarrow{J_{k}}$ vanishes on the boundary $\Sigma$.

Let us now discuss briefly the nature of these expressions for the entropy flow $\vec{J}_{s}$ and production rate $\sigma$, for they yield rather interesting observations.

Equation (2.20) tells us that the entropy flow into the volume has two contributions, one is the heat flux $\vec{J}_{q}$, as one expects, and the other is due to the diffusion flows of matter $\vec{J}_{k}$. Equation $(2.21)$ on the other hand shows that the entropy production rate is due to four terms: heat conduction, matter diffusion, gradients in the velocity field giving rise to viscous flow, and finally, chemical reactions.

The entropy production is in fact a bilinear form, since it is a sum of two-factor products (each linear in both arguments) of varying tensorial character. What is most interesting is that the factors belong to two distinct classes. The first type of quantities appearing in the expression for $\sigma$ is what De Groot and Mazur call a flow quantity [4] (chemical reaction rate $J_{j}$, viscous pressure tensor or momentum flows $\Pi$, heat flow $\vec{J}_{q}$, and diffusion flow $\overrightarrow{J_{k}}$ ), the reason for which is rather evident.

The other terms appearing in the bilinear form, those belonging to the second class, are gradients of intensive state variables (temperature, chemical potential, velocity), gradients of a potential energy in the form of the external force $\vec{F}_{k}$, and a linear combination of state variables $\mu_{k}$ in the expression for the affinity $A_{j}$. These quantities in scalar product with the various flows are usually called thermodynamic forces or affinities. What is key to understand is that, in general, entropy production rate can be expressed as a bilinear form combining thermodynamic fluxes and their respective "forces".

This observation will be invaluable in the next sections where we will postulate that the entropy production rate can in fact be always expressed as a bilinear form taking as arguments the various relevant flow quantities present in the system and their respective affinities. 


\section{Chapter 3}

\section{Linear Phenomenological Equations}

\subsection{Entropy Production and The Linear Laws}

Although we can garner qualitatively the meaning of the terms appearing in the entropy balance equation (2.20)-(2.21), the set of differential equations we need to solve are in a sense, empty. This is because they contain the irreversible fluxes (i.e. diffusion flows $\vec{J}_{k}$, heat flow $\vec{J}_{q}$, chemical reaction rates $J_{j}$ and momentum flow $\nabla \vec{v}$ ) as unknown quantities, so even if we specify the boundary and initial conditions of a given physical system, these equations cannot be solved.

To curb this issue we are required to construct, without a priori justification, a set of functional relations linking the unknown quantities together. Naturally the simplest functional form is the linear kind, so we introduce a set of equations known as the linear phenomenological laws, which allow us to express the unknown flux quantities as linear combinations of the thermodynamic forces (i.e. chemical affinity $A_{j}$, temperature gradient $\nabla T$, pressure tensor $\Pi$ and external forces $\vec{F}_{k}$ ). In this way we can reduce the number of degrees of freedom in the Cauchy problem (2.20-2.21 once we have specified the relevant boundary conditions) sufficiently to render it solvable, whether analytically or numerically.

Epistemologically speaking, this is the weak point of the theory of nonequilibrium thermodynamics that we're currently examining, in the sense that it this simplification which generally defines the limits of the theory. It is from this approximation that the title of linear irreversible thermodynamics stems from, and although stringent, it shouldn't come as a surprise. Many laws of this linear nature are known experimentally to be of great predictive power: Fourier's law of heat conduction, Fick's law of diffusion and Ohm's law of electric conduction. All of these laws require that the flux quantity in question (heat, particle diffusion, current) be expressed as the gradient of some corresponding quantity (temperature, particle concentration, electric potential) multiplied by a characteristic constant, to give rise to a solvable differential equation. 
Naturally, an argument from analogy of this kind is rather weak, so we shall need to verify a posteriori, through comparison with observational evidence, in what class of situations their use is justified.

At any rate we shall write the linear phenomenological equations in the following manner:

$$
J_{i}=\sum_{k} L_{i k} X_{k}
$$

The quantities $L_{i k}$ are called the phenomenological coefficients, while $J_{i}$ and $X_{i}$ are respectively the cartesian components of the independent fluxes and thermodynamic forces appearing in the expression for the entropy production rate $\sigma$ (2.21) we saw in the previous chapter:

$$
\begin{aligned}
\sigma & =\sum_{i} J_{i} X_{i} \\
\sigma & =\sum_{i} \sum_{k} L_{i k} X_{k} X_{i}
\end{aligned}
$$

Where in (3.3) we simply combined the linear phenomenological laws with (3.1). In order for the entropy production rate to be non-negative we clearly require that the matrix $L_{i k}$ is at least non-negative definite, since it defines uniquely the bilinear form (having chosen the canonical basis in $\mathbb{R}^{\mathrm{n}}$ ).

To include in this description the possibility of cross-phenomena such as thermal diffusion, i.e. diffusion flows depending on both concentrations and temperature gradients, we have also supposed in general that each individual flux is a linear combination of all the thermodynamic forces appearing in the entropy source strength.

While ordinary transport phenomena like heat conduction and electric conduction are linear in a vast number of cases, it is clearly possible to have irreversible phenomena which obey non-linear phenomenological laws (e.g. chemical reactions), and if such is the case, this present formulation cannot in general tackle them. Having said that, if the system is sufficiently near to equilibrium, the linear relations still function as an excellent approximation [4].

At any rate, it shall be implicitly supposed that henceforth, we shall only treat those phenomena which obey linear phenomenological laws. 


\subsection{The Curie Principle and Onsanger Reciprocal Relations}

In this section we deal with two of the most fundamental theorems in linear irreversible thermodynamics, the Curie Principle and the Onsanger Reciprocal Relations. These theorems, when applicable, simplify considerably the differential equations describing the evolution of the system's state [6] [3].

Since their proof is rather involved for a thesis of this length, we shall limit ourselves to their discussion only and not delve into their rigorous derivation.

\section{The Curie Principle}

Let $S$ be a system described locally by $N$ independent thermodynamic parameters and let the linear phenomenological laws hold as a relation between fluxes $\left(J^{s}, J^{v}, J^{T}\right)$ and thermodynamic forces $\left(X^{s}, X^{v}, X^{T}\right)$ :

$$
\begin{aligned}
& J^{s}=L^{s s} X^{s}+L^{s v} \cdot X^{v}+L^{s t}: X^{T} \\
& J^{v}=L^{v s} X^{s}+L^{v v} \cdot X^{v}+L^{v t}: X^{T} \\
& J^{T}=L^{t s} X^{s}+L^{t v} \cdot X^{v}+L^{t t}: X^{T}
\end{aligned}
$$

Where $X^{s}$ is a scalar (rank zero tensor), $X^{v}$ is a vector (rank one tensor), $X^{T}$ is a matrix (rank two), and the $J$ quantities have dimensions corresponding to their index as well. The $L$ quantities on the other hand correspond to linear applications and have rank determined by the dimension of its domain and codomain ${ }^{1}$.

If the state of the system is invariant under rotations, i.e. isotropic, then the fluxes and the thermodynamic forces of different tensorial character do not couple together [4], and the phenomenological laws (3.4)-(3.7) take the simpler form:

$$
\begin{aligned}
& J^{s}=L^{s s} X^{s} \\
& J^{v}=L^{v v} \cdot X^{v} \\
& J^{T}=L^{t t}: X^{T}
\end{aligned}
$$

\footnotetext{
${ }^{1}$ e.g. $L^{t t}$ is a rank four tensor, since its application to a rank two tensor yields a rank two tensor as a result.
} 
And so the expression for the entropy production rate will reduce to the sum of three independent product terms, involving only elements taken from the same vector space (of same dimensionality) and therefore three scalar products defined over $\mathbb{R}, \mathbb{R}^{3}$ and the space of $3 \times 3$ real matrices:

$$
\sigma=X^{s} L^{s s} X^{s}+X^{v} \cdot\left(L^{v v} \cdot X^{v}\right)+X^{T}:\left(L^{t t}: X^{T}\right)
$$

Where we assumed for simplicity that only one flow quantity of each tensorial rank is under consideration.

So, according to the Curie Principle if the system is isotropic then fluxes and forces of different tensorial ranks will not couple together in the phenomenological laws, and the entropy production will take the highly symmetric form (3.10).

Besides the patent simplifications this principle affords the problem, it also says something rather remarkable about the mechanisms by which entropy is produced within an isotropic system's volume element.

In an isotropic system, scalar fluxes such as the reaction rate are only caused by scalar thermodynamic forces like the chemical affinity, vectorial fluxes such as heat conduction are only due to vector forces such as temperature or concentration gradients, and the momentum flux, i.e. the pressure tensor, is only caused by rank two tensorial forces such as the gradient of the velocity field.

We only presented here the isotropic case, but other kinds of spatial symmetries can induce in the phenomenological equations other simplifications. Therefore, strictly speaking, the Curie Principle is much more general than the isotropic case. We only present here the isotropic case for brevity's sake, since this is the only case we shall need later on.

\section{The Onsanger Reciprocal Relations}

The Onsanger Reciprocal Relations, much like the Curie Principle, function as a further diminution of the redundant degrees of freedom in the phenomenological laws, through the symmetry given by the time reversal invariance of the equations of motion [4]. It is well known that in both quantum and classical mechanics, if no external magnetic field is present and the system is not rotating, the equations of motion in charge of the time evolution of the system are symmetric with respect to time. That is, the particles retrace their former path if all velocities have their sign reversed [6].

When time reversal symmetry is valid microscopically, Onsanger's reciprocal relations are valid macroscopically. [4] [6] As aforementioned, we shall simply state the content of this theorem.

We consider a non-rotating, adiabatically insulated system where no external magnetic field is present, described by $N=n+m$ independent parameters of two kinds, $\left(A_{1}, \ldots, A_{n}\right)$ and $\left(B_{1}, \ldots, B_{m}\right)$. The $A$ parameters are by definition even functions of 
the particle velocities (e.g. concentrations of chemical species, energy density, mass density) while the $B$ parameters are odd under reversal of the velocities $\vec{v} \mapsto-\vec{v}$ (e.g. momentum density). Since the system is adiabatically insulated, its entropy will rise due to irreversible processes occurring within, until it reaches equilibrium. We denote the equilibrium values of the defining parameters by $\left(A_{1}^{0}, \ldots, A_{n}^{0}\right)$ and $\left(B_{1}^{0}, \ldots, B_{m}^{0}\right)$.

The deviations of these parameters from their equilibrium values are then:

$$
\begin{gathered}
\alpha_{i}=A_{i}-A_{i}^{0} \\
\beta_{i}=B_{i}-B_{i}^{0}
\end{gathered}
$$

In first approximation, the deviation of entropy for a nonequilibrium state from its equilibrium value can be written as:

$$
\begin{gathered}
\Delta S=\Delta S(\vec{\alpha}, \vec{\beta}) \\
\Delta S=-\frac{1}{2} \sum_{i, k=1}^{n} G_{i k} \alpha_{i} \alpha_{k}-\frac{1}{2} \sum_{i, k=1}^{m} H_{i k} \beta_{i} \beta_{k}
\end{gathered}
$$

Where the matrices $G$ and $H$ constitute together the hessian matrix of $\Delta S$ split into two terms to distinguish the even and odd variables. Naturally both $G$ and $H$ are positive definite, since at equilibrium the entropy reaches a maximum.

Given that we have carried out a Taylor expansion in (3.14) we already supposed the system is sufficiently near to equilibrium such that this approximation be valid. Therefore, finding ourselves in a linear regime, we can further assume that the time behavior of the state parameters is described sufficiently well by a set of linear ordinary differential equations with constant coefficients [4]:

$$
\begin{array}{ll}
\frac{\mathrm{d} \alpha_{i}}{\mathrm{~d} t}=-\sum_{k=1}^{n} M_{i k}^{(\alpha \alpha)} \alpha_{k}-\sum_{k=1}^{m} M_{i k}^{(\alpha \beta)} \beta_{k} & i=1,2, \ldots, n \\
\frac{\mathrm{d} \beta_{i}}{\mathrm{~d} t}=-\sum_{k=1}^{n} M_{i k}^{(\beta \alpha)} \alpha_{k}-\sum_{k=1}^{m} M_{i k}^{(\beta \beta)} \beta_{k} & i=1,2, \ldots, m
\end{array}
$$


Onsanger's Theorem simplifies the problem by establishing relations of a symmetric nature between these coefficients, namely:

$$
\begin{aligned}
& \sum_{k=1}^{n} M_{i k}^{(\alpha \alpha)} G_{k j}^{-1}=\sum_{k=1}^{n} M_{j k}^{(\alpha \alpha)} G_{k i}^{-1} \quad i, j=1,2, \ldots, n \\
& \sum_{k=1}^{n} M_{i k}^{(\beta \beta)} H_{k j}^{-1}=\sum_{k=1}^{n} M_{j k}^{(\beta \beta)} H_{k i}^{-1} \quad i, j=1,2, \ldots, m \\
& \sum_{k=1}^{m} M_{i k}^{(\alpha \beta)} H_{k j}^{-1}=-\sum_{k=1}^{n} M_{j k}^{(\beta \alpha)} G_{k i}^{-1} \quad i=1,2, \ldots, n ; \quad j=1,2, \ldots, m
\end{aligned}
$$

Where $G^{-1}$ and $H^{-1}$ denote the inverse matrices of $G$ and $H$, which are always invertible given their positive definiteness (no eigenvalue of the hessian matrix is zero therefore neither is its determinant). At the moment these relations seem not to deal with the phenomenological coefficients $L_{i k}$ relating the thermodynamic forces and the fluxes in the linear equations (3.1), however we can recast them in a more transparent manner. To this effect we introduce the following variables, linear combinations of the deviations $\alpha_{i}$ and $\beta_{i}$, by partially deriving the expression for $\Delta S$.

$$
\begin{aligned}
& X_{i} \equiv \frac{\partial \Delta S}{\partial \alpha_{i}}=-\sum_{k=1}^{n} G_{i k} \alpha_{k} \quad i=1,2, \ldots, n \\
& Y_{i} \equiv \frac{\partial \Delta S}{\partial \beta_{i}}=-\sum_{k=1}^{m} H_{i k} \beta_{k} \quad i=1,2, \ldots, m
\end{aligned}
$$

Solving for the $\alpha_{i}$ and $\beta_{i}$ as a function of $X_{k}$ and $Y_{k}$ we obtain:

$$
\begin{array}{cc}
\alpha_{i}=-\sum_{k=1}^{n} G_{i k}^{-1} X_{k} & i=1,2, \ldots, n \\
\beta_{i}=-\sum_{k=1}^{m} H_{i k}^{-1} X_{k} & i=1,2, \ldots, m
\end{array}
$$


And combining these with the expressions for the rates of change of $\alpha_{i}$ and $\beta_{i}(3.15)$ and (3.16):

$$
\begin{array}{rl}
\frac{\mathrm{d} \alpha_{i}}{\mathrm{~d} t}=\sum_{k=1}^{n} L_{i k}^{(\alpha \alpha)} X_{k}+\sum_{k=1}^{m} L_{i k}^{(\alpha \beta)} Y_{k} & i=1,2, \ldots, n \\
\frac{\mathrm{d} \beta_{i}}{\mathrm{~d} t}=\sum_{k=1}^{n} L_{i k}^{(\beta \alpha)} X_{k}+\sum_{k=1}^{m} L_{i k}^{(\beta \beta)} Y_{k} & i=1,2, \ldots, m
\end{array}
$$

Where the coefficients are given by:

$$
\begin{aligned}
& L_{i k}^{(\alpha \alpha)}=\sum_{j=1}^{n} M_{i j}^{(\alpha \alpha)} G_{j k}^{-1} \quad i, k=1,2, \ldots, n \\
& L_{i k}^{(\alpha \beta)}=\sum_{j=1}^{m} M_{i j}^{(\alpha \beta)} H_{j k}^{-1} \quad i=1,2, \ldots, n \quad k=1,2, \ldots, m \\
& L_{i k}^{(\beta \alpha)}=\sum_{j=1}^{n} M_{i j}^{(\beta \alpha)} G_{j k}^{-1} \quad i=1,2, \ldots, m \quad k=1,2, \ldots, n \\
& L_{i k}^{(\beta \beta)}=\sum_{j=1}^{m} M_{i j}^{(\beta \beta)} H_{j k}^{-1} \quad i, k=1,2, \ldots, m
\end{aligned}
$$

In terms of these new quantities the Onsanger relations $(3.17)$ - (3.19) become:

$$
\begin{aligned}
& L_{i k}^{(\alpha \alpha)}=L_{k i}^{(\alpha \alpha)} \quad i, k=1,2, \ldots, n \\
& L_{i k}^{(\alpha \beta)}=-L_{k i}^{(\beta \alpha)} \quad i=1,2, \ldots, n \quad k=1,2, \ldots, m \\
& L_{i k}^{(\beta \beta)}=L_{k i}^{(\beta \beta)} \quad i, k=1,2, \ldots, m
\end{aligned}
$$

Where the symmetrical nature of the coefficients, inherited from the time-reversal symmetry of the equations of motion, is now fully manifest. To summarise, the Onsanger 
reciprocal relations are valid for the coefficients of the phenomenological equations if the independent "fluxes" $J_{i}$ and $I_{i}$ :

$$
\begin{aligned}
J_{i} \equiv \frac{\mathrm{d} \alpha_{i}}{\mathrm{~d} t} & i=1,2, \ldots, n \\
I_{i} \equiv \frac{\mathrm{d} \beta_{i}}{\mathrm{~d} t} & i=1,2, \ldots, m
\end{aligned}
$$

Are written as linear functions of the independent thermodynamic forces $X_{i}$ and $Y_{i}$, themselves partial derivatives of the entropy deviation $\Delta S$ with respect to $\alpha_{i}$ and $\beta_{i}$ :

$$
\begin{aligned}
X_{i} & =\frac{\partial \Delta S}{\partial \alpha_{i}} \quad i=1,2, \ldots, n \\
Y_{i} & =\frac{\partial \Delta S}{\partial \beta_{i}} \quad i=1,2, \ldots, m
\end{aligned}
$$

It is noteworthy, to make a full circle, to see how the derivative of $\Delta S$ with respect to time (i.e. the entropy production rate) looks like as a function of the fluxes $\left(I_{i}, J_{i}\right)$ and the thermodynamic forces $\left(X_{i}, Y_{i}\right)$.

Deriving (3.14) with respect to time one obtains:

$$
\frac{\mathrm{d} \Delta S}{\mathrm{~d} t}=-\sum_{i=1}^{n} \sum_{k=1}^{n} G_{i k} \alpha_{k} \frac{\mathrm{d} \alpha_{i}}{\mathrm{~d} t}-\sum_{i=1}^{m} \sum_{k=1}^{m} H_{i k} \beta_{k} \frac{\mathrm{d} \beta_{i}}{\mathrm{~d} t}
$$

Therefore with (3.20), (3.21), (3.33) and (3.34):

$$
\frac{\mathrm{d} \Delta S}{\mathrm{~d} t}=\sum_{i=1}^{n} J_{i} X_{i}+\sum_{i=1}^{m} I_{i} Y_{i}
$$

That is, the entropy production is a bilinear form of the fluxes and thermodynamic forces appearing in the phenomenological equations for which the Onsanger relations hold [4]. 
The Onsanger Relations tell us something physically extraordinary, which can easily get lost in translation when staring at their mathematical expression. For example, it is obvious to us already that when both a pressure gradient and a temperature gradient are present in a system, although one generally causes matter flows and the other heat flow, cross-phenomena are possible. That is, a temperature gradient on a surface of constant pressure can lead to matter flow (convection) and likewise, a pressure gradient at constant temperature can cause heat flow.

What Onsanger's Theorem therefore states, seemingly against all intuition, is that whenever this system is microscopically reversible, the heat flow per unit of pressure difference is precisely equal to the density flow per unit of temperature difference.

\subsubsection{Magnetic Fields and Rotating Systems}

We saw earlier that we excluded both rotating systems and ones in which an external magnetic field is present.

Magnetic fields are treated separately because when present, the sign of both the magnetic field $\vec{B}$ and the velocity $\vec{v}$ need to be reversed in order for the Lorentz force $\vec{F}=q(\vec{E}+\vec{v} \times \vec{B})$ to remain invariant, and for microscopic reversibility to hold.

For rotating systems, the case for the angular velocity is analogous to the magnetic field, given the expression of the Coriolis force contains a $\vec{v} \times \vec{\omega}$ term.

In light of these considerations, when either of these situations occur, the Onsanger relations need to be modified in the following manner [4]:

$$
\begin{aligned}
& L_{i k}^{(\alpha \alpha)}(\vec{B}, \vec{\omega})=L_{k i}^{(\alpha \alpha)}(-\vec{B},-\vec{\omega}) \quad i, k=1,2, \ldots, n \\
& L_{i k}^{(\alpha \beta)}(\vec{B}, \vec{\omega})=-L_{k i}^{(\beta \alpha)}(-\vec{B},-\vec{\omega}) \quad i=1,2, \ldots, n \quad k=1,2, \ldots, m \\
& L_{i k}^{(\beta \beta)}(\vec{B}, \vec{\omega})=L_{k i}^{(\beta \beta)}(-\vec{B},-\vec{\omega}) \quad i, k=1,2, \ldots, m
\end{aligned}
$$

Where, in line with conventional notation, $\vec{B}$ stands for the external magnetic field and $\vec{\omega}$ is the angular velocity.

The Onsanger relations in a sense "break down" when external magnetic fields or angular velocities are present, for we can only relate the coefficients together in two distinct physical situations, one in which the external magnetic field or the angular velocity are oriented a certain way in space and one in which they point in the opposite direction (their norm obviously kept fixed). 


\section{Chapter 4}

\section{Application to Thermoelectric Phenomena}

\subsection{The Seebeck, Peltier and Thompson Effects}

We now turn our attention to an application of what we have learned, to see if correct predictions can be made by using linear irreversible thermodynamics as our starting point.

In particular, we shall derive L. Kelvin's Relations for Thermoelectric Phenomena, first discovered by W. Thompson in 1851, they express the functional relationship obeyed by the Seebeck, Peltier and Thompson coefficients, driving properties of their respective effects [9].

Thermoelectric effects occur whenever temperature gradients in a thermocouple induce the flow of electric current or the formation of an electric potential (Seebeck Effect); whenever a current driven through the junction of two different conductors at the same temperature leads to cooling or heating of the junction (Peltier Effect); whenever a current driven through a conductor with a temperature gradient leads to cooling or heating of the conductor (Thompson Effect) [6].

\section{The Seebeck Effect}

The Seebeck Effect was discovered by by J. Seebeck in 1821 when he noticed his compass needle deflect in the vicinity of a closed circuit composed of two different metal filaments $(A$ and $B$ ), whose junctions he had heated to different temperatures ( $T$ and $T+\Delta T)$.

The temperature gradient present in the circuit thus constructed (known as a thermocouple) had induced an electromotive force, allowing current to flow around the closed loop and so producing a magnetic field capable of interfering with Seebeck's compass. 
Figure 4.1: Schematic picture of a Thermocouple.

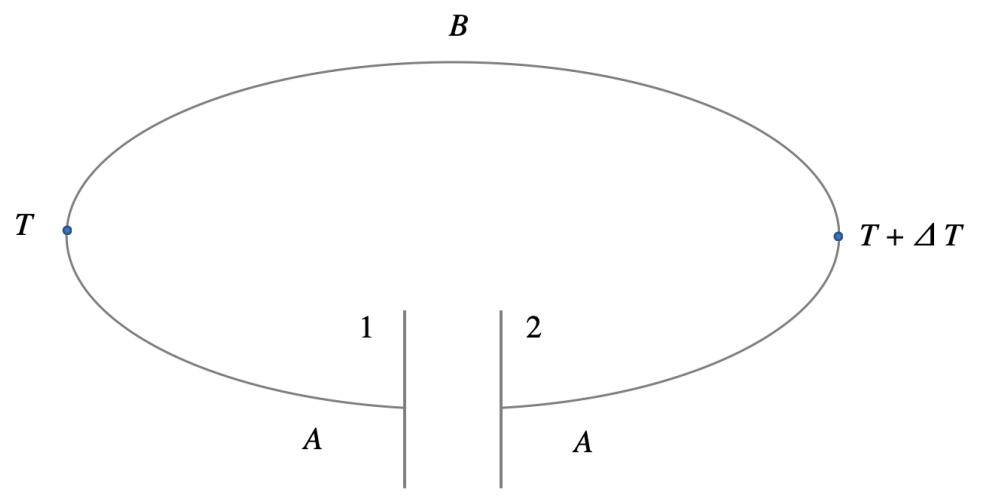

Seebeck concluded that once the system had reached a stationary state (i.e. no current), the difference in electric potential $\Delta \phi$ across the conducting plates 1 and 2 induced by the temperature gradient was directly proportional to the difference in temperature [8], where the proportionality coefficient $S_{A B}$ is now known as the Seebeck coefficient ${ }^{1}$ :

$$
\begin{array}{r}
\Delta \phi=S_{A B} \Delta T \\
S_{A B}=\lim _{\Delta T \rightarrow 0} \frac{\Delta \phi}{\Delta T}
\end{array}
$$

\section{The Peltier Effect}

The Peltier Effect, discovered empirically by Jean-Charles Peltier in 1834, occurs when a current flows across a junction of two different conductors. Peltier observed that heat that could not be accounted for only by Joule heating, was being removed or added at the junction ${ }^{2}$.

Referring once more to Figure 4.1 of a generic thermocouple, we place a potential difference generator across the condenser plates, giving rise to a current of intensity $i$ through the circuit. The two junctions are kept at the same temperature, and the heat expended at the junctions is measured over time.

The heat $\Delta Q$ produced at the junction per unit time (not due to Joule heating) obeys the following expression, discovered empirically by Peltier himself [7]:

\footnotetext{
${ }^{1}$ The Seebeck coefficient is a characteristic property of the types of conductors used, hence the index $A B$, and although usually treated as a constant, its value varies with temperature.

${ }^{2}$ The junction is heated if the current flows in a certain direction, and removed if the current flows the opposite way.
} 


$$
\begin{aligned}
\frac{\Delta Q}{\Delta t} & =\pi_{A B} i \\
\pi_{A B} & =\lim _{\Delta t \rightarrow 0} \lim _{i \rightarrow 0} \frac{1}{i} \frac{\Delta Q}{\Delta t}
\end{aligned}
$$

Where $\pi_{A B}$ is the Peltier coefficient, a property of the junction of metals $A$ and $B$ quantifying the amount of heat absorbed (if $\pi_{A B}$ is positive) or removed (if $\pi_{A B}$ is negative) per unit of time per unit of electric current.

At this point an important remark should be made on the Seebeck and Peltier coefficients. Both the Seebeck and Peltier effects do not occur if thermocouple is entirely composed of a single conducting material. The difference in the physical properties between metals $A$ and $B$ gives rise to a discontinuity in the circuit which proves essential, without which these thermoelectric phenomena are not present.

This leads us to believe that the Peltier and Seebeck coefficients $\pi_{A B}$ and $S_{A B}$ need to vanish at a junction composed of the same metal, since with all else remaining constant in expressions (4.1) and (4.3), the left hand-side vanishes identically.

So although we have defined these coefficients operationally, and not independently of metals $\mathrm{A}$ and $\mathrm{B}$ forming a junction together, it is reasonable to suppose that each material is characterised by its own coefficients. Furthermore at a junction of two different metals the discontinuity giving rise to these effects is simply encoded in $\pi_{A B}$ and $S_{A B}$ in the following manner:

$$
\begin{array}{ccc}
\pi_{A B}=\pi_{A}-\pi_{B} & \Rightarrow & \pi_{A A}=\pi_{B B}=0 \\
S_{A B}=S_{A}-S_{B} & \Rightarrow & S_{A A}=S_{B B}=0
\end{array}
$$

As we shall see, the Seebeck and Peltier effects are inextricably linked, and represent different manifestations of the same physical reality. A fact discovered by Lord Kelvin and proved by Lars Onsanger with the help of his reciprocal relations almost a century later, it became an important landmark for the theory of linear irreversible thermodynamics.

Before we move to that however, we shall complete the triptych and discuss briefly the Thompson Effect in thermoelectricity.

\section{The Thompson Effect}


In $1851 \mathrm{~W}$. Thompson (Lord Kelvin) produced a treatise called "On a mechanical theory of thermo-electric currents", where he delved into the recent discoveries made by Peltier and Seebeck and predicted, alongside the Peltier and Seebeck Effects, a possible third phenomenon, now aptly named the Thompson Effect.

The Thompson effect or Thompson heating occurs when an electric current flows in a temperature gradient through a conductor. Heat unaccounted for by Joule heating is either released or absorbed by the conductor, once more depending on the direction of current flow with respect to the temperature gradient. Lord Kelvin predicted that for a current of density $\vec{I}$ flowing through a conductor along a gradient $\nabla T$, a heat production rate per unit volume $\dot{q}$ would evolve at a point $x$ of the conductor according to:

$$
\dot{q}(x)=\tau \vec{I}(x) \cdot \nabla T(x)
$$

Where $\tau$ is the Thompson coefficient, and in general is a function of both position (along the conductor) and temperature ${ }^{3}$. Physically, the Thompson coefficient quantifies the amount of heat evolved per unit of current density per unit of time per unit of temperature gradient, in the infinitesimal volume around a particular point in the conductor $[9]$.

Unlike the Seebeck and Peltier effects, the Thompson effect can arise in a single conductor, so long as a temperature gradient is present and a current is forced through it, so we can immediately attribute a Thompson coefficient to each conducting material.

\section{The Thompson Relations}

In 1854, Thompson would elaborate further on the subject of thermoelectric phenomena in his seminal work "On a Dynamical Theory of Heat". In this text he found relationships linking the coefficients of the three effects discussed so far: The Peltier, Seebeck and Thompson effects. Thompson discovered that all three coefficients were expressible in terms of a single one, thus demonstrating how all three effects were in fact different variations of a single underlying interplay between electric currents, temperature gradients and discontinuities in the makeup of the system [10]:

\footnotetext{
${ }^{3}$ For most purposes, when using a homogeneous linear conductor and in certain intervals of temperatures $\tau$ is assumed to be constant in value.
} 


$$
\begin{aligned}
& \text { (i) } \quad \tau=-\frac{\partial \pi}{\partial T}-S \\
& \text { (ii) } \pi=-T S
\end{aligned}
$$

Using (4.8) in conjunction with (4.9) we can in fact express the Thompson coefficient as a function of temperature and the Seebeck coefficient alone:

$$
\tau=T \frac{\partial S}{\partial T}
$$

\subsection{Proof of Thompson's Relations}

In all of the thermoelectric effects listed above, the system in question is patently in a nonequilibrium state. Thompson was therefore one of the first physicists to delve into the description of nonequilibrium systems, and was able to prove the first of his relations (4.8) using only the $1^{\text {st }}$ Law of Thermodynamics [10]. He however was not able to prove the second of his relations starting from first principles, and rather was forced to make an additional assumption regarding "reversible" contributions to the process:

"The electromotive forces produced by inequalities of temperature in a circuit of different metals, and the thermal effects of electric current circulating in it, are subject to the laws which would follow from the general principles of the thermodynamic theory of heat if there were no conduction of heat from one part of the circuit to another."[10]

Thompson treated this statement as a conjecture, its validity needing to be verified through experiment. Essentially a reflection on the spectral nature of the Peltier and Seebeck effects, how one is the mirror-image of the other and how each could be reversed in its effect (direction of current induced in Seebeck, the inversion of heated and cooled junctions in Peltier) by simply reversing the experimental setup, would lead to the induced symmetry between the Peltier coefficient and the Seebeck coefficient we see in (4.9) His seemingly reasonable assumption was that these effects marked the reversible contributions to the thermodynamic transformations the system was undergoing ${ }^{4}$. Boltzmann himself attempted to prove that this assumption was actually a consequence of more fundamental principles in thermodynamics, but we now know that no basis for this principle exists [4].

\footnotetext{
${ }^{4}$ The irreversible contributions happening alongside would be the flow of heat due to Fourier's law of conduction and Joule heating.
} 
L. Onsager was the first to prove that it is not so-called "reversible contributions" to the process, but rather the assumption of microscopic reversibility, which lead to Thomspon's second relation $\pi=-T S[6]$. In fact the reciprocal relations, which we saw stated in general terms in chapter 3 , once applied to the instance of a thermocouple will lead trivially to the proof of Thompson's relations.

\section{Irreversible Thermodynamics of a Thermocouple}

The proof will employ all the theoretical background work we so far have developed, and therefore the assumptions made in its construction will be required to hold. The system is locally in equilibrium ${ }^{5}$ and microscopically reversible, since no external magnetic field is present and since the laws of motion are time reversible. The linear phenomenological laws hold since no chemical reactions are occurring [4] (in general a nonlinear function of the thermodynamic forces) and since, as aforementioned, the linear laws of heat and electrical conduction (Fourier and $\mathrm{Ohm}$ ) remain valid in a large class of physical situations.

We begin our proof with the expression of the entropy production rate in a slightly different form. Since of little didactic worth, the derivation is given in Appendix A.2:

$$
T \sigma=-\vec{J}_{s, t o t} \cdot \nabla T-\vec{I} \cdot\left[\nabla\left(\frac{\mu_{e}}{z_{e}}\right)-\vec{E}\right]
$$

Where $\mu_{e}$ is the chemical potential of the charge carriers and $z_{e}$ is their charge, $\vec{E}$ is the electric field, $\vec{I}$ is the electric current density and $\vec{J}_{s, t o t}$ is equal to the entropy flux density $\vec{J}_{s}$ plus the convective term $\rho s \vec{v}$, where the velocity $\vec{v}$ is the velocity of the charge carriers as measured in the reference frame given by the metal's ion lattice.

From inspection of the fluxes $\left(\vec{J}_{s, t o t}, \vec{I}\right)$ and thermodynamic forces $\left(\nabla T, \nabla\left(\frac{\mu_{e}}{z_{e}}\right)-\vec{E}\right)$ present in (4.11), we can immediately write the linear phenomenological equations (3.1) as:

$$
\begin{array}{r}
\vec{J}_{s, t o t}=-L_{11} \nabla T-L_{12}\left[\nabla\left(\frac{\mu_{e}}{z_{e}}\right)-\vec{E}\right] \\
\vec{I}=-L_{21} \nabla T-L_{22}\left[\nabla\left(\frac{\mu_{e}}{z_{e}}\right)-\vec{E}\right]
\end{array}
$$

\footnotetext{
${ }^{5}$ Temperature, electric and chemical potential are well-defined locally and differentiable functions of the space coordinates.
} 
Where we restricted our discussion to only isotropic systems, so that we may use Curie's Principle as it was presented in this paper.

The Onsanger relation (3.30) immediately tells us that:

$$
L_{21}=L_{12}
$$

We can rewrite (4.12) and (4.13) in a more transparent form by solving for $\vec{J}_{s, t o t}$ and $\vec{E}-\nabla\left(\frac{\mu_{e}}{z_{e}}\right)$, yielding, after some reinterpreting of the phenomenological coefficients as constants we already know the physical meaning of:

$$
\begin{gathered}
\vec{J}_{s, t o t}=-\frac{k}{T} \nabla T+\frac{\pi}{T} \vec{I} \\
\vec{E}-\nabla\left(\frac{\mu_{e}}{z_{e}}\right)=-S \nabla T+R \vec{I} \\
\pi=-T S
\end{gathered}
$$

Where $k$ is the thermal conductivity of the medium for vanishing current, $R$ is the isothermal resistivity, $\mathrm{S}$ is called the differential thermoelectric power, we shall see why momentarily, [4] and has dimensions of an electric field per unit of temperature gradient, and finally $\frac{\pi}{T}$, the entropy transported at constant temperature per unit time and unit of electric current. Equation (4.17) is a direct consequence of the Onsanger relation $L_{12}=L_{21}$ and the demonstration is trivial, one needs simply compare coefficients in the expressions for $\vec{J}_{s, t o t}$ and $\vec{E}-\nabla\left(\frac{\mu_{e}}{z_{e}}\right)$ in terms of the phenomenological coefficients and those appearing in (4.15) and (4.16).

Simply from dimensional considerations and comparison with the expressions for the Seebeck and Peltier effects (4.1) and (4.3), it is reasonable to suppose that $\pi$ and $S$ are in fact the Peltier and Seebeck coefficients. This is in fact the case and thus justifies our use of notation.

To prove the statement that these are in fact the Peltier and Seebeck coefficients we turn our attention once more to the thermocouple (Figure 4.1). Essentially, a thermocouple consists of two homogeneous metal wires of different kinds, $A$ and $B$ connected together at two junctions $j_{1}$ and $j_{2}$, with a condenser with plates 1 and 2 placed in one of the wires, let's say $A$. Each junction is placed in its own heat bath so as to ensure constant temperature at the respective junction, but with $j_{1}$ at temperature $T$ and $j_{2}$ at temperature $T+\Delta T$. As we saw earlier, this is analogous to the setup Seebeck employed.

We are now interested in calculating the difference in electric potential $\Delta \phi$ across the plates of the condenser at the stationary state of zero electric current $\vec{I}=0$. We 
know, from Maxwell's equations, that $\vec{E}=-\nabla \phi-\frac{\partial \vec{A}}{\partial t}$. That is, that the electric field is equal to the gradient of the electric potential $\phi$ and the partial time derivative of the vector potential $\vec{A}$. However, given that the system arrives at a steady-state, the vector potential $\vec{A}$ does not depend on time explicitly. The derivative term then vanishes and $\nabla \phi=-\vec{E}$.

With this in mind the potential difference across the plates is simply given by the line integral:

$$
\begin{aligned}
& \Delta \phi=\phi_{1}-\phi_{2}=\int_{2}^{1} \nabla \phi \cdot \overrightarrow{d l}=\int_{1}^{2} \vec{E} \cdot \overrightarrow{d l} \\
& \Delta \phi=\int_{1}^{2} \nabla\left(\frac{\mu_{e}}{z_{e}}\right) \cdot \overrightarrow{d l}-\int_{1}^{2} S \nabla T \cdot \overrightarrow{d l}
\end{aligned}
$$

Where in (4.19) we have expressed the electric field using (4.16) with $\vec{I}=0$.

Now, the first integral on the right hand side is equal to zero, since the chemical potential is a function of temperature, pressure, and electron concentration only. The temperature is held constant across the condenser plates by the experimental setup, the pressure is equal given the system finds itself in mechanical equilibrium and is electroneutral $(z=0)$, and the concentration of electrons on the plates is virtually the same [4].

Therefore the chemical potential is constant across the plates, and the first line integral is zero.

As to the second term on the right hand side, we assume that the coefficient $S$ in each homogeneous metal wire $A$ and $B$ remains sufficiently constant as to be taken outside the integral sign, even though for very large temperature differences it is in fact variable. So we break up the remaining integral in (4.19) and readily obtain the same result obtained, via experimental means, by Seebeck:

$$
\begin{aligned}
& \Delta \phi=\int_{1}^{j_{1}}-S \nabla T \cdot \overrightarrow{d l}+\int_{j_{1}}^{j_{2}}-S \nabla T \cdot \overrightarrow{d l}+\int_{j_{2}}^{2}-S \nabla T \cdot \overrightarrow{d l} \\
& \Delta \phi=-S_{A}\left[T\left(j_{1}\right)-T(1)\right]-S_{B}\left[T\left(j_{2}\right)-T\left(j_{1}\right)\right]-S_{A}\left[T(2)-T\left(j_{2}\right)\right] \\
& \Delta \phi=\left(S_{A}-S_{B}\right) \Delta T=S_{A B} \Delta T
\end{aligned}
$$

Where we have labelled $j_{1}$ the first junction one encounters moving from plate 1 to plate 2 around the circuit depicted in Figure 4.1, and $j_{2}$ the other remaining junction. In 
addition we remembered that the condenser plates are at the same temperature $T(2)=$ $T(1)$ by construction and therefore the two terms containing them cancel each other out.

We now consider the Peltier effect, and in particular the quantification of Peltier heat, defined as the heat absorbed/released at a junction between two metals $A$ and $B$ per unit of current across the junctions connecting them. To this end we employ the complete expression for the entropy balance equation (2.20) and (2.21) we derived in the chapter 2, but applied to the present case. As we saw then, the balance equation can be written as:

$$
\rho \frac{\mathrm{d} s}{\mathrm{~d} t}=-\overrightarrow{J_{s}}+\sigma
$$

Given our expression for the entropy production rate $\sigma$ for thermoelectric effects is (4.11), we can explicitly write the balance equation as:

$$
\rho \frac{\mathrm{d} s}{\mathrm{~d} t}=-\nabla \cdot \vec{J}_{s}-\frac{1}{T} \vec{J}_{s, t o t} \cdot \nabla T-\frac{1}{T} \vec{I} \cdot\left[\nabla\left(\frac{\mu_{e}}{z_{e}}\right)-\vec{E}\right]
$$

Or alternatively, by using relation (1.16) as:

$$
\frac{\partial \rho s}{\partial t}=-\nabla \cdot \vec{J}_{s, t o t}-\frac{1}{T} \vec{J}_{s, t o t} \cdot \nabla T-\frac{1}{T} \vec{I} \cdot\left[\nabla\left(\frac{\mu_{e}}{z_{e}}\right)-\vec{E}\right]
$$

And after introducing the phenomenological equations (4.15) and (4.16) and the Onsanger relation (4.17) we obtain:

$$
\frac{\partial \rho s}{\partial t}=\frac{1}{T} \nabla \cdot(k \nabla T)-\nabla \cdot \frac{\pi \vec{I}}{T}+\frac{R \vec{I}^{2}}{T}
$$

The first term represents entropy change due to heat conduction and has the wellknown form of Fourier's law, the second term we shall see is directly related to the Peltier effect, while the third is the expression of Joule heating in a linear conductor (derived from Ohm's law) divided by the temperature.

Now, let us consider an experimental setup identical to the one in Figure 4.1 of thermocouple, save for the fact that the conductor has been replaced by a potential 
difference generator such that current is allowed to flow around the closed circuit, and only junction $j_{1}$ is placed in a heat reservoir. At uniform temperature an electric current is flowing from metal $A$ to metal $B$.

We suppose that junction $j_{1}$ where metal $A$ and $B$ meet is represented by an infinitesimal volume $V$ of cross sections $\Sigma_{A}$ and $\Sigma_{B}$. We can imagine $V$ as essentially two adjacent cylinders, since the surface areas of $\Sigma_{A}$ and $\Sigma_{B}$ needn't be equal in general.

Integrating now expression (4.26) over the volume $V$ overlapping $j_{1}$ and remembering that $j_{1}$ is at constant temperature throughout (since in contact with a heat reservoir of fixed temperature) we obtain the expression for the rate of entropy change in this region $V$ :

$$
T \frac{\mathrm{d} S_{V}}{\mathrm{~d} t}=-\int_{V} \nabla \cdot \pi \vec{I} \mathrm{~d} V+\int_{V} R \vec{I}^{2} \mathrm{~d} V
$$

Whereupon, applying Gauss' divergence theorem and treating $\pi$ as a constant whose value depends only on the kind of conductor:

$$
T \frac{\mathrm{d} S_{V}}{\mathrm{~d} t}=\pi_{A} \int_{\Sigma_{A}} \vec{I} \cdot \mathrm{d} \vec{\Sigma}_{A}-\pi_{B} \int_{\Sigma_{B}} \vec{I} \cdot \mathrm{d} \vec{\Sigma}_{B}+\int_{V} R \vec{I}^{2} \mathrm{~d} V
$$

Where $\pi_{A}$ and $\pi_{B}$ are the values coefficient $\pi$ takes in the pure metals $A$ and $B$ and both $\vec{\Sigma}_{A}$ and $\vec{\Sigma}_{B}$ are counted positive in the direction of $\vec{I}$. Now, in the limit $V \rightarrow 0$ ( $j_{1}$ is reduced to a single surface) the volume integral on the right-hand side of (4.28) vanishes, since both the resistivity $R$ and the current density $I$ remain finite and the cross sections $\Sigma_{A}$ and $\Sigma_{B}$ tend to the common surface $\Sigma$ of the junction.

We thus find, applying the limit $V \rightarrow 0$, that the entropy rate of change for a discontinuous metal junction through which current flows is:

$$
T \frac{\mathrm{d} S}{\mathrm{~d} t}=\left(\pi_{A}-\pi_{B}\right) \int_{\Sigma} \vec{I} \cdot \mathrm{d} \vec{\Sigma}=\left(\pi_{A}-\pi_{B}\right) i
$$

Where we have labelled $i$ the current intensity flowing through $j_{1}$.

To support the steady state in which the junction finds itself (constant temperature), the change in entropy must be compensated by absorbing/releasing heat from/into the reservoir, giving thus rise to the Peltier effect [4]. In fact, calling $\pi_{A B}$ the Peltier heat we defined earlier as the amount of heat per unit time per unit current being absorbed/released at the junction, we have that: 


$$
\begin{aligned}
\pi_{A B} & \equiv \frac{T}{i} \frac{\mathrm{d} S}{\mathrm{~d} t}=\pi_{A}-\pi_{B} \\
\dot{Q} & =\left(\pi_{A}-\pi_{B}\right) i
\end{aligned}
$$

Remembering the empirically deduced relation due to Peltier (4.3), it is clear that (4.30) is completely equivalent.

We have therefore, without ambiguity, identified the phenomenological coefficients $\pi$ and $S$ as being the same ones appearing in the empirical Peltier and Seebeck equations. Recalling then the Onsanger relation (4.16), we obtain the exact expression of Thompson's second relation between the thermoelectric power and the Peltier heat, proving its validity:

$$
\pi_{A B}=-T S_{A B}
$$

Lastly, let us examine in detail the Thompson effect and prove Thompson's first relation (4.8). As we discussed, Thompson heat occurs when a current flows in a temperature gradient. Similarly to the Peltier effect, the entire physics of Thompson heat is contained in the entropy balance equation (4.26). We can rewrite the second term on the right hand side of that equation by applying the Leibniz rule of differentiation:

$$
-\nabla \cdot \frac{\pi \vec{I}}{T}=-\frac{\vec{I}}{T} \cdot \nabla \pi+\frac{\pi \vec{I}}{T^{2}} \cdot \nabla T
$$

Where we remembered to apply the stationarity condition

$$
\nabla \cdot \vec{I}=0
$$

Now, since the Peltier coefficient is a function of both position and temperature, we can write:

$$
\begin{gathered}
\pi=\pi(x, y, z, T)=\pi(x, y, z, T(x, y, z)) \rightarrow \\
\left.\nabla \pi=\left[\left(\frac{\partial \pi}{\partial x}\right)_{T}+\frac{\partial \pi}{\partial T} \frac{\partial T}{\partial x},\left(\frac{\partial \pi}{\partial y}\right)_{T}+\frac{\partial \pi}{\partial T} \frac{\partial T}{\partial y},\left(\frac{\partial \pi}{\partial z}\right)_{T}+\frac{\partial \pi}{\partial T} \frac{\partial T}{\partial z}\right)\right] \\
\nabla \pi=(\nabla \pi)_{T}+\frac{\partial \pi}{\partial T} \nabla T
\end{gathered}
$$


Upon inserting (4.34) into (4.33) one obtains:

$$
-\nabla \cdot \frac{\pi \vec{I}}{T}=-\frac{\vec{I}}{T} \cdot(\nabla \pi)_{T}-\left(\frac{\partial \pi}{\partial T}-\frac{\pi}{T}\right) \frac{\vec{I}}{T} \cdot \nabla T
$$

From which we can immediately see that the term splits into two parts: the first part tells us that a heat term is present even in the absence of a temperature gradient (Peltier). The second clearly defines a heat transport due to the mere presence of a current in a temperature gradient, and is therefore the term corresponding to the Thompson effect.

The Thompson coefficient $\tau$ is then naturally (comparing with (4.7) for the sign):

$$
\tau=\frac{\pi}{T}-\frac{\partial \pi}{\partial T}=-S-\frac{\partial \pi}{\partial T}
$$

Where we have used (4.17). This concludes the proof of Thompson's two relations for thermoelectric phenomena. 


\section{Conclusion}

In this treatise, albeit limited in scope, we have attempted to extend the laws of thermodynamics to systems not in equilibrium, but which maintain nonetheless a local notion of it. We have seen how in order to construct a coherent theory along these mesoscopic lines, the thermodynamic parameters used to describe the system's state locally, need to possess a certain degree of regularity. The system cannot therefore occupy states arbitrarily far from equilibrium.

Moreover, we have described how the balance equation for the entropy rate of change per unit volume is derived and how it constitutes, in this regime of applicability, an extension to the second law of thermodynamics, and how for a closed system we recover a generalized version of the Clausius-Carnot theorem. We have discussed the necessity of introducing the linear phenomenological laws in expressing the flows as linear combinations of the thermodynamic forces in order to obtain a solvable set of differential equations for the system's evolution. Their existence cannot always be justified a priori but we have seen how, if chemical reactions (flows which in general are nonlinear functions of the thermodynamic forces) are not present, the constraint on the system not being arbitrarily far from equilibrium is compatible with this postulate.

We completed the theoretical picture by stating two important theorems in linear irreversible thermodynamics, due to Curie and Onsanger, which simplify considerably the computational side of solving the balance equations. The Curie principle exploits the system's spatial symmetries to limit the manner in which thermodynamic forces of different tensorial rank can couple together in the phenomenological laws. Concomitantly, the Onsager relations take advantage of the time-reversal symmetries of the equations of motions (quantum or classical) in microscopically reversible systems to show that the matrix of phenomenological coefficients is composed of symmetric and antisymmetric blocks, thus expressing profound relations among experimentally measurable quantities.

Finally, we saw an example of the application of the theory to the well-known thermoelectric effects discovered by Seebeck, Peltier and Lord Kelvin (Thompson). From the entropy balance equation and the phenomenological laws we discussed and derived in this paper, we were able to recover the experimental results obtained by the same Seebeck, Peltier and Kelvin. Something which had already been achieved by Thompson already in 1854, however not from first principles. The Onsanger relations in the meantime, 
immediately and effortlessly, proved Kelvin's second relation between the thermoelectric power and the Peltier heat, a proof which, before the advent of Onsanger's relations, had proved impossible to carry out in a rigorous manner. 


\section{Appendix A.1}

We seek to prove that the entropy production rate $\sigma$ is invariant under galilean transformations, that is, transformations of the form

$$
\vec{v}_{k} \quad \rightarrow \quad \vec{v}_{k}+\vec{V}
$$

Where as usual $\vec{v}_{k}$ is the velocity of the $k^{t h}$ chemical component with respect to an arbitrary inertial reference frame and $\vec{V}$ is a constant vector. As we already discussed, it is necessary that $\sigma$ be invariant for Galilean transformations, since a thermodynamic transformation should seem irreversible irrespective of the observer (inertial). Naturally we suppose, in considering Galilean and not Lorentz transformations, that the velocities of all relevant bodies are small compared to the speed of light, and thus the problem reduces to the non-relativistic case.

Rewriting the expression for the entropy production rate (2.21) here for convenience:

$$
\sigma=-\frac{1}{T^{2}} \vec{J}_{q} \cdot \nabla T-\frac{1}{T} \sum_{k=1}^{n} \vec{J}_{k} \cdot\left[T \nabla\left(\frac{\mu_{k}}{T}\right)-\vec{F}_{k}\right]-\frac{1}{T} \Pi: \nabla \vec{v}-\frac{1}{T} \sum_{j=1}^{r} A_{j} J_{j}
$$

We can take each term one by one and see how they change under (A.1.1):

$$
\begin{aligned}
& \vec{v} \quad \rightarrow \quad \vec{v}^{\prime}=\sum_{k} \frac{\rho_{k}}{\rho}\left(\vec{v}_{k}+\vec{V}\right)=\sum_{k} \frac{\rho_{k}}{\rho} \vec{v}_{k}+\vec{V}=\vec{v}+\vec{V} \\
& \vec{J}_{k} \rightarrow \vec{J}_{k}^{\prime}=\rho_{k}\left(\vec{v}_{k}^{\prime}-\vec{v}^{\prime}\right)=\rho_{k}\left(\vec{v}_{k}+\vec{V}-\vec{v}-\vec{V}\right)=\rho_{k}\left(\vec{v}_{k}-\vec{v}\right)=\vec{J}_{k} \\
& \vec{F}_{k}^{\prime}=-\nabla \psi_{k}^{\prime}=-\nabla \psi_{k}=\vec{F}_{k}
\end{aligned}
$$

The other terms in the expression for $\sigma$ do not depend on the particular frame of

reference being used. Substituting the above into (A.1.1) and recalling that $\vec{V}$ is constant, i.e. $\nabla V=0$, one finds that:

$$
\sigma^{\prime}=\sigma
$$

That is, if a thermodynamic process appears reversible to a particular inertial observer $(\sigma=0)$ so will it for every possible inertial observer, making irreversibility an observerindependent notion in linear irreversible thermodynamics. 


\section{Appendix A.2}

We seek to prove that the entropy production rate

$$
\sigma=-\frac{1}{T^{2}} \vec{J}_{q} \cdot \nabla T-\frac{1}{T} \sum_{k=1}^{n} \vec{J}_{k} \cdot\left[T \nabla\left(\frac{\mu_{k}}{T}\right)-\vec{F}_{k}\right]-\frac{1}{T} \Pi: \nabla \vec{v}-\frac{1}{T} \sum_{j=1}^{r} A_{j} J_{j}
$$

can be expressed, for an isotropic, chemically inert, mechanically equilibrated system immersed in an electric field, as:

$$
T \sigma=-\vec{J}_{s, t o t} \cdot \nabla T-\vec{I} \cdot\left[\nabla\left(\frac{\mu_{e}}{z_{e}}\right)-\vec{E}\right]
$$

We suppose first of all that The force $\vec{F}_{k}$ a charged particle feels in the presence of an electric field $\vec{E}$ and a magnetic field $\vec{B}$ is the Lorentz force:

$$
\vec{F}_{k}=z_{k}(\vec{E}+\vec{v} \times \vec{B})
$$

Where $z_{k}$ is the charge per unit mass of the $k^{t h}$ chemical element in the system and $\vec{v}$ is velocity with respect to the reference frame in which the external magnetic field is stationary.

Since

$$
\overrightarrow{J_{k}}=\rho_{k}\left(\vec{v}_{k}-\vec{v}\right)
$$

Then one has that

$$
\vec{F}_{k}=z_{k}\left(\vec{E}+\vec{v}_{k} \times \vec{B}\right)-\frac{z_{k}}{\rho_{k}}\left(\vec{J}_{k} \times \vec{B}\right)
$$

Meaning that

$$
\vec{J}_{k} \cdot \vec{F}_{k}=z_{k} \vec{J}_{k} \cdot\left(\vec{E}+\vec{v}_{k} \times \vec{B}\right)-\frac{z_{k}}{\rho_{k}} \vec{J}_{k} \cdot\left(\overrightarrow{J_{k}} \times \vec{B}\right)=z_{k} \vec{J}_{k} \cdot\left(\vec{E}+\vec{v}_{k} \times \vec{B}\right)
$$

Since $\vec{J}_{k}$ is orthogonal to $\overrightarrow{J_{k}} \times \vec{B}$ always. 
Substituting (A.2.5) into (A.2.1) yields:

$$
T \sigma=-\frac{1}{T} \vec{J}_{q} \cdot \nabla T-\sum_{k=1}^{n} \vec{J}_{k} \cdot\left[T \nabla\left(\frac{\mu_{k}}{T}\right)-z_{k}\left(\vec{E}+\vec{v}_{k} \times \vec{B}\right)\right]
$$

Whereupon, substituting in

$$
T \nabla\left(\frac{\mu_{k}}{T}\right)=-\frac{\mu_{k}}{T} \nabla T+\nabla \mu_{k}
$$

into (A.2.6)

$$
T \sigma=-\frac{1}{T} \vec{J}_{q} \cdot \nabla T+\sum_{k=1}^{n} \frac{\mu_{k}}{T} \vec{J}_{k} \cdot \nabla T-\sum_{k=1}^{n} \vec{J}_{k} \cdot\left[\nabla \mu_{k}-z_{k}\left(\vec{E}+\vec{v}_{k} \times \vec{B}\right)\right]
$$

We recall that we defined the entropy flux density $\vec{J}_{s}$ as

$$
\overrightarrow{J_{s}}=\frac{1}{T}\left(\overrightarrow{J_{q}}-\sum_{k=1}^{n} \mu_{k} \overrightarrow{J_{k}}\right)
$$

In terms of the entropy flux density, (A.2.7) becomes

$$
T \sigma=-\vec{J}_{s} \cdot \nabla T-\sum_{k=1}^{n} \vec{J}_{k} \cdot\left[\nabla \mu_{k}-z_{k}\left(\vec{E}+\vec{v}_{k} \times \vec{B}\right)\right]
$$

Now we apply a theorem, due to Prigogine, which states that if the system is at mechanical equilibrium (as we supposed ours is), then the barycentric velocity $\vec{v}$ occurring in the definition of the diffusion flow $\vec{J}_{k}$, can be replaced by an arbitrary velocity $\vec{v}^{a}$ [4].

(A.2.8) thus reads:

$$
T \sigma=-\left[\vec{J}_{s}+\rho s\left(\vec{v}-\vec{v}^{a}\right)\right] \cdot \nabla T-\sum_{k=1}^{n} \vec{J}_{k}^{a} \cdot\left[\nabla \mu_{k}-z_{k}\left(\vec{E}+\vec{v}_{k} \times \vec{B}\right)\right]
$$

Where

$$
\vec{J}_{k}^{a}=\rho_{k}\left(\vec{v}_{k}-\vec{v}^{a}\right)
$$

And applying once more equation (A.2.5) one gets:

$$
T \sigma=-\left[\vec{J}_{s}+\rho s\left(\vec{v}-\vec{v}^{a}\right)\right] \cdot \nabla T-\sum_{k=1}^{n}{\overrightarrow{J_{k}}}^{a} \cdot\left[\nabla \mu_{k}-z_{k}\left(\vec{E}+\vec{v}^{a} \times \vec{B}\right)\right]
$$


This is an incredibly useful and general form for systems in mechanical equilibrium, for it allows us, for a particular choice of $\vec{v}^{a}$ to eliminate a degree of freedom, anyway redundant, from our differential equation. In particular, we choose

$$
\vec{v}^{a}=\vec{v}_{n}
$$

That is the velocity of the $n^{\text {th }}$ chemical component. This $n^{\text {th }}$ component could be the positive ion lattice of a metal, or the neutral solvent in an electrolytic solution. In that case, we can simplify the problem considerably by choosing a reference system in which these components are at rest

$$
\vec{v}^{a}=\vec{v}_{n}=0
$$

The fluxes then become simply

$$
\vec{J}_{k}^{a} \equiv \vec{J}_{k}^{r}=\rho_{k} \vec{v}_{k}
$$

And expression (A.2.10) becomes

$$
T \sigma=-\vec{J}_{s, t o t} \cdot \nabla T-\sum_{k=1}^{n-1} \vec{J}_{k}^{r} \cdot\left(\nabla \mu_{k}-z_{k} \vec{E}\right)
$$

Specialising finally to the case of a metal, as needed in the treatment of the thermocouple, we shall a single type of particle involved, the electron, with the positive ion lattice remaining by definition immobile. All fluxes will be measured relative to such a lattice and constitute therefore a perfect model for electron transport in a metal.

$$
T \sigma=-\vec{J}_{s, t o t} \cdot \nabla T-\vec{J}_{e}^{r} \cdot\left(\nabla \mu_{e}-z_{e} \vec{E}\right)
$$

At last, defining our current density as

$$
\vec{I}=z_{e} \vec{J}_{e}^{r}
$$

We obtain our desired result, (A.2.2):

$$
T \sigma=-\vec{J}_{s, t o t} \cdot \nabla T-\vec{I} \cdot\left[\nabla\left(\frac{\mu_{e}}{z_{e}}\right)-\vec{E}\right]
$$

Thus concluding our proof. 


\section{Bibliography}

[1] R. Clausius, The Mechanical Theory of Heat with its Applications to the SteamEngine and to the Physical Properties of Bodies, London: John van Voorst (1867).

[2] F. H. Crawford, Heat, Thermodynamics and Statistical Physics, Harcourt, Brace \& World (1963).

[3] P. Curie, Sur la Symétrie dans les Phénomènes Physiques, Symétrie d'un Champ Électrique et d'un Champ Magnétique" [On the Symmetries of Physical Phenomena, the Electric Field, and the Magnetic Field], Journal de Physique Théorique et Appliquée (1894).

[4] S. R. De Groot, P. Mazur, Non-Equilibrium Thermodynamics, North-Holland Publishing Company (1962).

[5] E. Fermi, Thermodynamics, Dover Publications (1956).

[6] L. Onsanger, Reciprocal Relations in Irreversible Processes I, Physical Review, American Physical Society (APS) (1931).

[7] J.-C. Peltier, Nouvelles Expériences sur la Caloricité des Courants Èlectrique [New Experiments on the Heat Effects of Electric Currents], Annales de Chimie et de Physique (1834).

[8] J. Seebeck, Magnetische Polarisation der Metalle und Erze durch TemperaturDifferenz [Magnetic polarization of metals and ores by temperature differences], Abhandlungen der Königlichen Akademie der Wissenschaften zu Berlin (1822).

[9] W. Thompson, On a Mechanical Theory of Thermo-Electric Currents, Proceedings of the Royal Society of Edinburgh (1851).

[10] W. Thompson, On the Dynamical Theory of Heat. Part V: Thermo-Electric Currents, Transactions of the Royal Society of Edinburgh (1854). 